\title{
Caractérisation non destructive de la zone endommagée des galeries souterraines excavées dans les roches argileuses
}

\author{
Philippe Cosenza ${ }^{1, \star}$, Stephen Hedan ${ }^{1}$ et Valéry Valle ${ }^{2}$ \\ ${ }^{1}$ Université de Poitiers, IC2MP-HydrASA, UMR CNRS 7285, École nationale supérieure d'ingénieurs (ENSI) de Poitiers, \\ 1, rue Marcel-Doré, TSA 41105, 86073 Poitiers cedex 09, France \\ ${ }^{2}$ Université de Poitiers, CNRS, UPR 3346, Institut PPRIME, SP2MI, boulevard Marie-et-Pierre-Curie, Téléport 2, BP 30179,86962 \\ Futuroscope Chasseneuil cedex, France
}

\begin{abstract}
Résumé - Cet article passe en revue les différentes méthodes utilisées pour la caractérisation et le suivi non destructifs de la zone endommagée des ouvrages excavés dans les roches argileuses. Trois familles de méthodes sont identifiées. La première famille qui relève de la géophysique appliquée inclut la sismique réfraction et l'analyse de la dispersion des ondes de surface. Ces méthodes permettent d'avoir rapidement et avec un dispositif plutôt léger une quantification de l'épaisseur de la zone endommagée. La deuxième famille vise à obtenir une représentation $2 \mathrm{D}$, voire $3 \mathrm{D}$, de cette zone endommagée. Elle comprend les méthodes sismiques «puits à puits» (tomographie sismique notamment) et la tomographie électrique. Les développements méthodologiques récents relatifs à ces méthodes permettent d'envisager à court terme un suivi 4D en promouvant une inversion de type time-lapse des données acquises. Ces méthodes sont toutefois plus lourdes à mettre en œuvre et l'interprétation des données nécessite des temps de calcul importants et des outils numériques spécifiques et délicats à manipuler pour un non-géophysicien. Les méthodes optiques de corrélation relèvent d'une troisième famille de méthodes. Elles ont surtout l'avantage d'obtenir directement et avec une très haute résolution des grandeurs mécaniques (cinématiques) associées à la fissuration de surface. Les résultats des expérimentations menées avec ces trois familles de méthodes en galeries souterraines (laboratoire souterrain du Mont-Terri, station expérimentale de Tournemire et laboratoire souterrain de Meuse/Haute-Marne) sont également présentés et brièvement discutés.
\end{abstract}

Mots clés : méthodes non destructives / méthodes géophysiques / méthodes optiques de corrélation / zone endommagée / roches argileuses

\begin{abstract}
Non-destructive characterization of the damaged zone of underground galleries excavated in clay rocks. This paper reviews the different methods used to characterize and to monitor in a nondestructive way the excavation damaged zone in clay rocks. Three groups of methods are identified. The first group is constituted by geophysical methods: the refraction seismic method and the analysis of surface waves method. These methods allow to quickly obtain the thickness of the damage zone with a rather simple system of acquisition. The second group of methods aims to obtain a 2D or even 3D model of the damaged zone. This group includes "well-to-well" seismic methods (e.g., seismic tomography) and electrical resistivity tomography. The recent methodological developments associated with these methods allow to consider at short term 4D monitoring by promoting "time-lapse" inversion. However, using of these methods is rather cumbersome: the corresponding data processing can be time-consuming and uses specific numerical tools which are difficult to handle for non-geophysicists. Digital image correlation (DIC) methods belong to the third group of methods. They have the advantage of obtaining directly and with a high resolution some kinematic parameters associated with the surface cracking observed on the gallery walls. The results obtained from these three groups of methods and acquired in three underground laboratories (Mont-Terri laboratory, experimental station of Tournemire and Meuse/Haute-Marne laboratory) are also presented and briefly discussed.
\end{abstract}

Keywords: non-destructive methods / geophysical methods / digital image correlation methods / excavated damaged zones / clay rocks

\footnotetext{
^Auteur de correspondance : philippe.cosenza@univ-poitiers.fr
} 


\section{Introduction}

De nombreux pays industriels (notamment la Belgique, le Canada, les États-Unis, la France, le Japon, la Suisse) examinent actuellement l'intérêt du stockage géologique profond de leurs déchets radioactifs. Parmi les formations géologiques envisagées, les formations argileuses constituent des candidates intéressantes pour au moins deux raisons. D'une part, elles sont connues pour posséder une très faible conductivité hydraulique (typiquement inférieure à $10^{-12} \mathrm{~m} \cdot \mathrm{s}^{-1}$ ). D'autre part, elles présentent des propriétés de rétention significatives qui pourraient être mobilisées pour l'adsorption de radionucléides qui migreraient dans la formation géologique.

Toutefois, l'excavation d'infrastructures souterraines induit l'apparition d'une zone endommagée en paroi des galeries. Cette zone endommagée qui résulte de la redistribution des contraintes dans la roche constitue une zone fracturée plus ou moins importante dont l'extension dépend de la technique de creusement utilisée, de la géométrie de la galerie (forme, diamètre, etc.), du contexte géologique (e.g., orientation de l'axe de la galerie versus l'orientation de la stratification, état de contrainte initial), des propriétés pétrophysiques (porosité, teneur en eau, conductivité hydraulique) et bien évidemment du comportement hydromécanique de la roche (Blümling et al., 2007). Dans le cas particulier des roches argileuses étudiées aujourd'hui dans quatre laboratoires souterrains (laboratoire de Meuse/Haute-Marne de l'Agence nationale pour la gestion des déchets radioactifs, ANDRA en France ; station expérimentale de Tournemire en France ; laboratoire souterrain du Mont-Terri en Suisse ; laboratoire souterrain de Mol en Belgique), les observations de la zone endommagée en paroi et à partir de carottes ont permis de mettre en évidence des fractures associées aux modes de rupture suivants : extension, cisaillement, voire mixte (extension et cisaillement) (Bossart et al., 2002 ; Tsang et al., 2005 ; Blümling et al., 2007 ; Armand et al., 2014). Il est important ici de souligner que les fractures observées sont bien associées à des facturations consécutives du seul creusement des galeries. Nous renvoyons les lecteurs intéressés aux articles de Blümling et al. (2007) et d'Armand et al. (2014) pour des descriptions plus détaillées. En outre, les mesures au laboratoire et in situ ont montré que la conductivité hydraulique de la roche argileuse dans la zone endommagée augmentait de plusieurs ordres de grandeur (au moins de quatre ordres de grandeur sur le site du laboratoire de Meuse/HauteMarne : de $10^{-8}$ à $10^{-12} \mathrm{~m} . \mathrm{s}^{-1}$, e.g., Baechler et al., 2011). Cette zone endommagée pourrait donc influencer négativement les performances de confinement du stockage géologique.

Par conséquent, la caractérisation du comportement à court et à long termes de cette zone endommagée dans les roches argileuses est devenue un enjeu essentiel pour la sûreté des stockages géologiques réalisés dans ces formations. Une des options possibles pour mener à bien cette caractérisation est aujourd'hui d'utiliser des méthodologies non destructives et non invasives qui pourraient être mises en œuvre directement dans les laboratoires souterrains. En effet, ces méthodes offrent au moins deux avantages. D'une part, elles fournissent une information spatialement bien échantillonnée compte tenu de leur rapidité de mise en œuvre. D'autre part, certaines d'entre elles permettent d'estimer indirectement et quantitativement des paramètres précieux (porosité, variation de teneur en eau, etc.) à partir d'un calibrage ad hoc sur des échantillons carottés et/ou à partir d'approches théoriques spécifiques. Comme mentionné plus loin, ces méthodologies relèvent en majorité de méthodes de la géophysique appliquée. Toutefois, d'autres méthodes, notamment les méthodes optiques de corrélations qui permettent d'accéder directement à des champs cinématiques, peuvent aussi être envisagées.

L'objectif de cet article de revue est de présenter un état de l'art des méthodologies non destructives utilisées pour la caractérisation et le suivi de la zone endommagée présente en paroi des ouvrages excavés dans les roches argileuses. Il est divisé en quatre parties. Dans une première partie, la démarche générale et les principes qui sous-tendent la mise en œuvre de ces méthodes sont présentées. Puis, la deuxième et la troisième parties exposent et discutent les résultats obtenus respectivement par les méthodes sismiques et électriques. La dernière partie montre les développements récents de méthodes optiques de corrélation dans le contexte particulier de l'étude de la zone endommagée des galeries souterraines.

\section{Méthodologie générale}

\subsection{Déroulement d'une campagne de mesures}

Comme pour toutes les méthodes géophysiques, une campagne de mesures acquises par une ou des méthodes non destructives comporte les cinq étapes successives suivantes (e.g., Lagabrielle, 1998) :

- caractérisation de la cible : cette étape importante permet d'identifier les méthodes les plus pertinentes au regard de la problématique posée. Cette étape qui vise également à acquérir de l'information a priori sur la cible est discutée plus en détail dans la prochaine section ;

- définition des paramètres d'acquisition : il s'agit essentiellement de définir la géométrie du dispositif d'acquisition et les pas d'échantillonnage (temps, espace) de la ou des méthode(s) identifiée(s) à l'étape précédente ;

- la campagne d'acquisition de données ;

- le traitement géophysique des données acquises ;

- l'interprétation des données : l'objectif de cette étape est de proposer in fine un modèle statique ou dynamique de la cible envisagée et plus largement du (sous-)terrain exploré

Nous renvoyons les lecteurs intéressés par ces aspects plus méthodologiques aux ouvrages de Mari et al. (1998), Lagabrielle (1998) et surtout au document intitulé « Géophysique appliquée. Code de bonne pratique » disponible sur le site internet de l'association professionnelle AGAP-Qualité (1992).

\subsection{Caractérisation de la cible}

Il s'agit essentiellement au cours de cette étape de définir l'extension géométrique de la cible (profondeur, taille/ dimension) des méthodes non destructives employées et les contrastes de propriétés physiques associés à la cible identifiée. En effet, suivant ce deuxième objectif, la cible doit constituer une zone d'anomalie de la propriété physique mise en jeu dans la méthode utilisée, c'est-à-dire une zone dans laquelle la valeur de la propriété physique va contraster significativement avec celle de l'encaissant. 


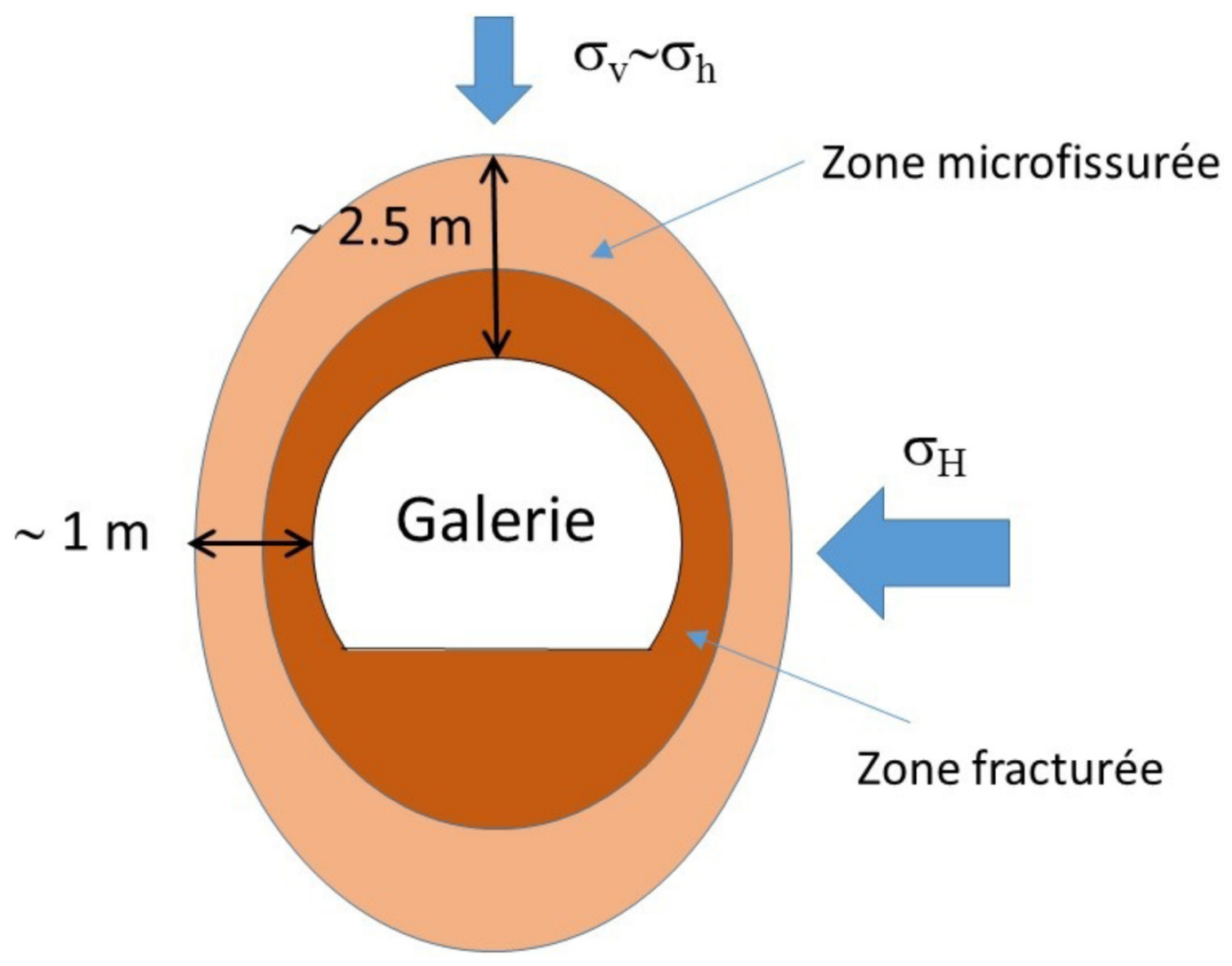

Fig. 1. Modèle conceptuel de la zone endommagée d'une galerie d'environ $2 \mathrm{~m}$ de rayon et parallèle à la direction de la contrainte principale horizontale mineure, $\sigma_{\mathrm{h}}$, dans le laboratoire de Meuse/Haute-Marne (modifié de Baechler et al., 2011).

Fig. 1. Conceptual model of the excavated dammed zone around a 2-m radius gallery, parallel to $\sigma_{h}$ in the Meuse/Haute-Marne Underground Research Laboratory (modified from Baechler et al., 2011).

Dans notre contexte, la zone endommagée des galeries souterraines constitue naturellement la cible à privilégier. Mais, d'autres cibles plus localisées, i.e., une fracture ou un groupe de fractures observées en paroi peuvent être également envisagées notamment lorsque des méthodes plus locales, des méthodes optiques par exemple, sont utilisées. En effet, dès lors que la zone endommagée met en évidence des fractures débouchant en paroi des galeries, l'utilisation de méthodes optiques qui permettent non seulement de spatialiser des champs cinématiques sur les parements mais aussi de quantifier l'ouverture/fermeture des fractures apparaît également intéressante.

La plupart des mesures obtenues par des méthodes destructives s'accorde pour décrire la zone endommagée comme une enveloppe à deux épaisseurs entourant la galerie (Fig. 1). L'enveloppe interne délimitée par la paroi de la galerie constitue une zone dense de fractures. Toutefois, des levés géologiques récents identifient une organisation plus complexe de la zone endommagée qui prendrait la forme d'une fracturation en chevrons (Armand et al., 2014 ; La Vaissière et al., 2015). La profondeur de la zone endommagée est estimée de l'ordre d'un rayon (Bossart et al., 2002 ; Tsang et al., 2005), voire de deux rayons, de galerie suivant le contexte géomécanique, i.e., suivant l'orientation des directions des contraintes principales vis-à-vis de la direction de l'axe de la galerie (Baechler et al., 2011 ; La Vaissière et al., 2015). Les mesures au laboratoire indiquent également que deux familles de propriétés pétrophysiques sont sensibles à la fracturation et donc à la désaturation associée de la roche argileuse : les vitesses des ondes élastiques (par exemple, Sarout et Guéguen, 2008 ; Ghorbani et al., 2009) et la résistivité électrique (par exemple, Cosenza et al., 2007 ; Ghorbani, 2007) (Fig. 2). En effet, il convient de garder à l'esprit que la zone endommagée résulte non seulement de processus mécaniques mais, dans le cas particulier des roches argileuses, reste associée à des phénomènes hydromécaniques complexes (i.e., chute de la pression de pores et désaturation de la roche pouvant induire retrait et fissuration).

Finalement, l'ensemble des caractéristiques géométriques et pétrophysiques des cibles envisagées et des méthodes non destructives associées est résumé dans le Tableau 1. Cette synthèse permet de dégager trois familles de méthodes pertinentes : les méthodes sismiques, la tomographie électrique et les méthodes optiques qui seront abordées dans la suite de cet article. Il convient ici de souligner que les méthodes électromagnétiques en hautes fréquences, de type radar géologique, souvent utilisées en reconnaissance géotechnique sont peu adaptées en contexte argileux : les ondes électromagnétiques en hautes fréquences sont fortement atténuées dans les matériaux riches en minéraux argileux. 


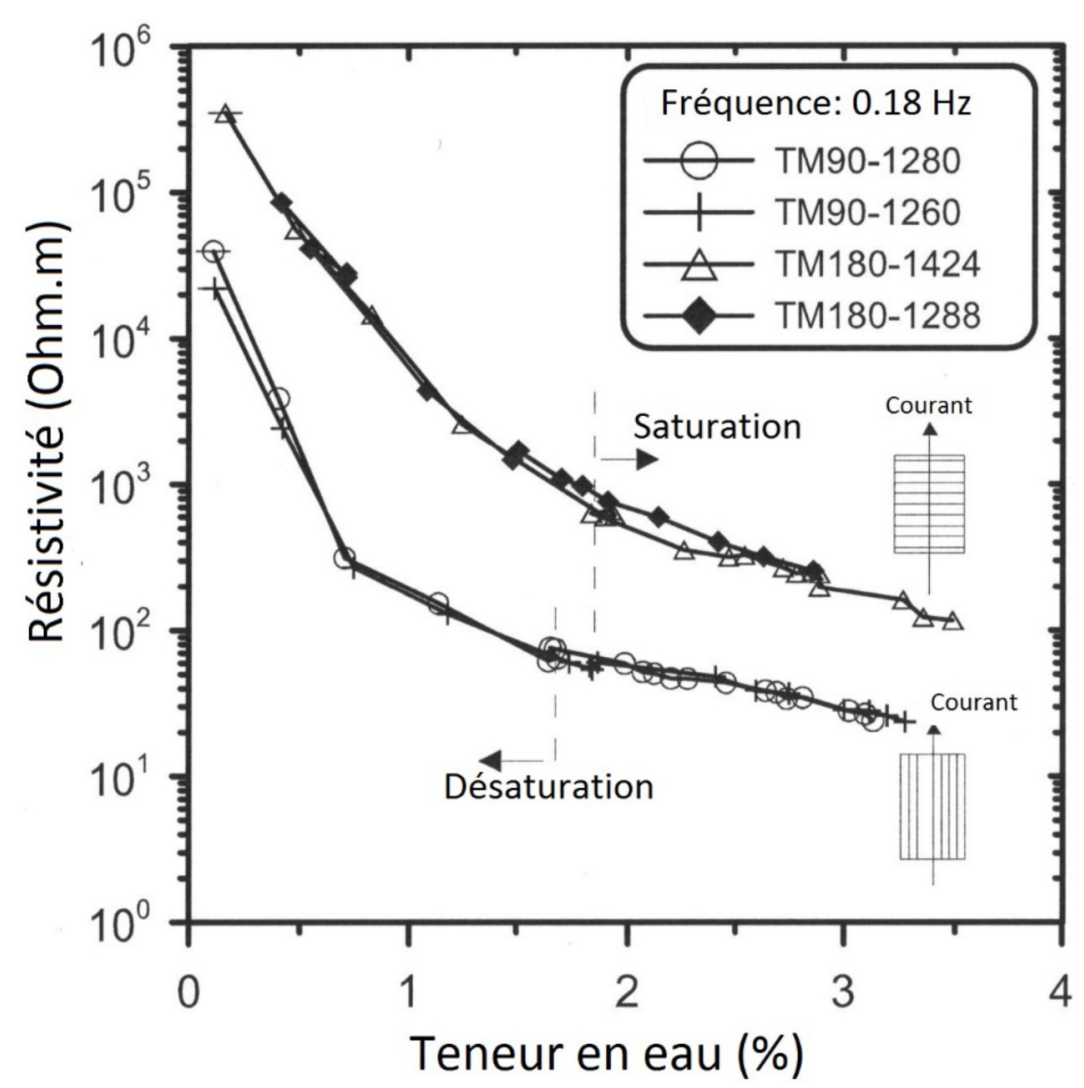

Fig. 2. Résistivité en fonction de la teneur en eau d'échantillons d'argilite de Tournemire. Deux directions du courant électriques sont considérées : une direction perpendiculaire à la stratification et une direction parallèle à la stratification (Ghorbani, 2007).

Fig. 2. Resistivity as a function of water content of Tournemire argillite samples. Two directions of electrical current are considered: one direction perpendicular to bedding and one parallel to bedding (Ghorbani, 2007).

Tableau 1. Méthodes non destructives utilisées pour la caractérisation de la zone endommagée.

Table 1. Non-destructive methods used for the characterization of the damaged zone.

\begin{tabular}{llll}
\hline Cible & Méthode & Grandeur mesurée & Paramètre physique \\
\hline $\begin{array}{l}\text { L'ensemble de } \\
\text { la zone } \\
\text { endommagée }\end{array}$ & $\begin{array}{l}\text { Sismique } \\
\text { Émission } \\
\text { acoustique } \\
\text { Électrique }\end{array}$ & $\begin{array}{l}\text { Temps de trajet } \\
\text { ou amplitude } \\
\text { champ physique }\end{array}$ & $\begin{array}{l}\text { Origine du } \\
\text { mécaniques ou } \\
\text { atténuation }\end{array}$ \\
$\begin{array}{l}\text { Groupe de fractures } \\
\text { observées en paroi }\end{array}$ & Optique & $\begin{array}{l}\text { Différence de potentiel } \\
\text { électrique }\end{array}$ & Résistivité \\
& Déplacement & $\begin{array}{l}\text { Champs cinématiques } \\
\text { (champs de déplacement, } \\
\text { tenseur des déformations) }\end{array}$ \\
\hline
\end{tabular}

\section{Méthodes sismiques}

\subsection{Généralités}

Les méthodes sismiques sont basées sur l'étude de la propagation d'ondes mécaniques dans le géomatériau investigué. Les vitesses de propagation des ondes impliquées dépendent des modules élastiques et de la masse volumique du géomatériau.
Plusieurs types d'ondes se propagent simultanément : les ondes de volumes (ondes de compression dites ondes $\mathrm{P}$ et ondes de cisaillement dites ondes $\mathrm{S}$ ) se distinguent des ondes de surface qui ne se propagent qu'au voisinage des interfaces séparant deux géomatériaux de propriétés élastiques et de masse volumique différente (voir par exemple, Mari et al., 1998 ; Lagabrielle, 1998). Dans le cadre de travaux menés sur des roches argileuses soumises à un endommagement, il est important de souligner que deux aspects sont souvent considérés. Le premier 


\section{a)}
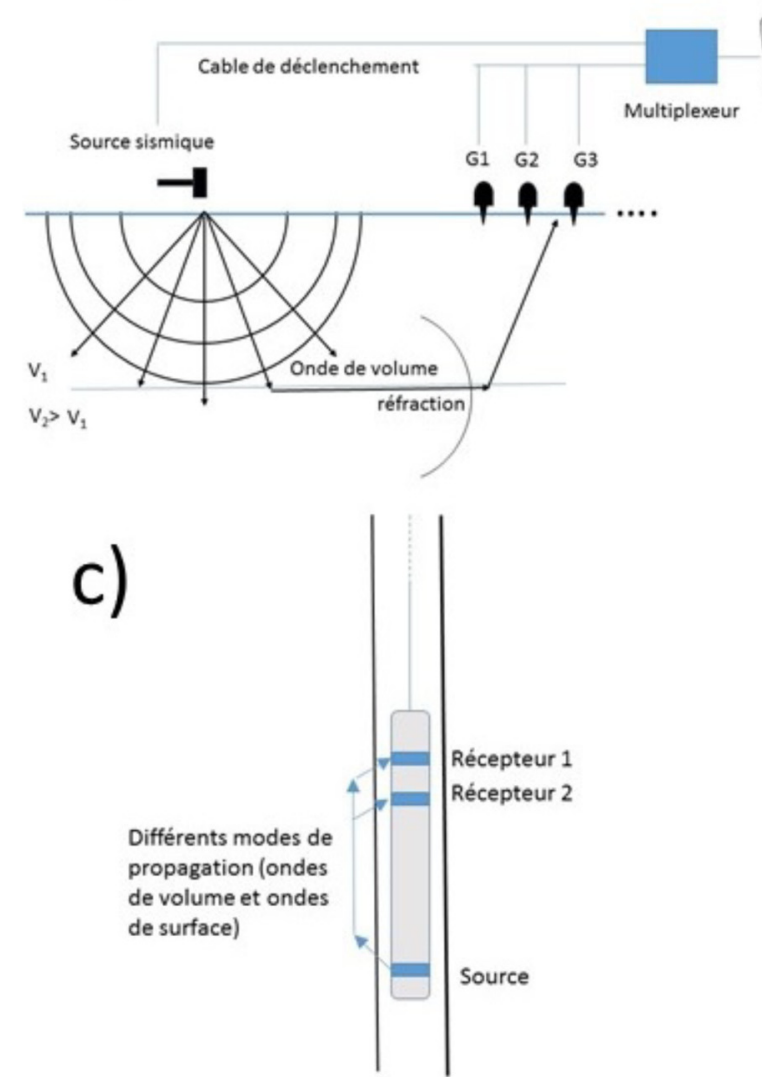

b)
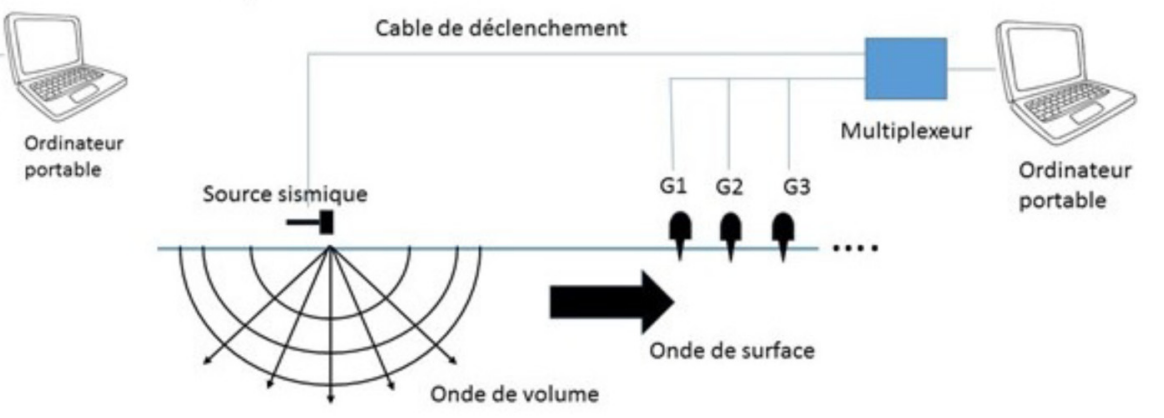

d)

Fig. 3. Les différentes méthodes sismiques utilisées pour la caractérisation et le suivi de la zone endommagée : a : la sismique réfraction ; b : l'analyse des ondes de surface ; $\mathrm{c}:$ la diagraphie sismique ; $\mathrm{d}:$ les méthodes sismiques « puits à puits ».

Fig. 3. Different seismic methods used to characterize and to monitor the excavation damaged zone: a: the seismic refraction method; $b$ : the analysis of surface waves; $c$ : the seismic logging; $d$ : the "well-to-well" seismic methods.

aspect porte sur l'effet de la désaturation de la roche, consécutive à l'excavation. L'onde $\mathrm{S}$ ne se propageant pas dans les fluides (et donc insensible aux variations de teneur en eau du matériau), l'étude combinée des vitesses des ondes $\mathrm{P}$ et $\mathrm{S}$ permet alors de discriminer l'effet de la seule désaturation. Toutefois, en pratique, comme le montreront les études présentées plus loin, la mesure de la vitesse d'onde $\mathrm{S}$ a été introduite très récemment dans les galeries souterraines. Le deuxième aspect concerne la nature isotrope transverse de la roche argileuse : les propriétés physiques de cette dernière sont symétriques par rotation autour de l'axe perpendiculaire à la stratification (les plans de stratification constituent alors des plans de symétrie : toutes les propriétés sont identiques dans toutes directions comprises dans ces plans). Comme pour les mesures des ondes $\mathrm{S}$, l'anisotropie mécanique de la roche argileuse a été abordée récemment et fait encore l'objet d'intensives recherches (e.g., Delle Piane et al., 2015).

Dans notre contexte, les méthodes sismiques qui ont prouvé leur efficacité pour l'étude spécifique de la zone endommagée autour de galeries souterraines sont, d'une part, les méthodes sans forage de type sismique réfraction et utilisation de la dispersion des ondes de surface et, d'autre part, celles qui nécessitent un ou plusieurs forages : diagraphie sismique et méthodes sismiques dites de « puits à puits » (Fig. 3). Toutes ces méthodes sont présentées dans la suite de cet article. Cette revue se limitera toutefois aux méthodes dites « actives », c'est-àdire utilisant une source sismique, i.e., un dispositif permettant l'émission d'ondes sismiques. L'approche passive par émission acoustique qui résulte d'un dégagement d'énergie sous forme d'ondes élastiques accompagnant le processus d'endommagement ne sera pas présentée : l'atténuation relativement élevée des ondes sismiques dans les milieux argileux rend délicate l'emploi de cette méthode et les résultats obtenus ont été généralement moins probants (Le Gonidec et al., 2012).

À ce stade, il est aussi important de souligner que les méthodes ici mises en œuvre ont souvent utilisé des équipements spécifiques et/ou ont fait l'objet de développements technologiques particuliers (e.g., Pettitt et al., 2004) : miniaturisation des capteurs; méthodologies de couplage capteur-roche, utilisation de source sismique de très haute fréquence (plusieurs dizaines de kilohertz) et de récepteurs permettant d'enregistrer des signaux dans des bandes de fréquences élevées (typiquement plusieurs dizaines de kilohertz) ; conception de chaîne d'acquisition permettant des fréquences d'échantillonnage élevées (supérieures au mégahertz). 


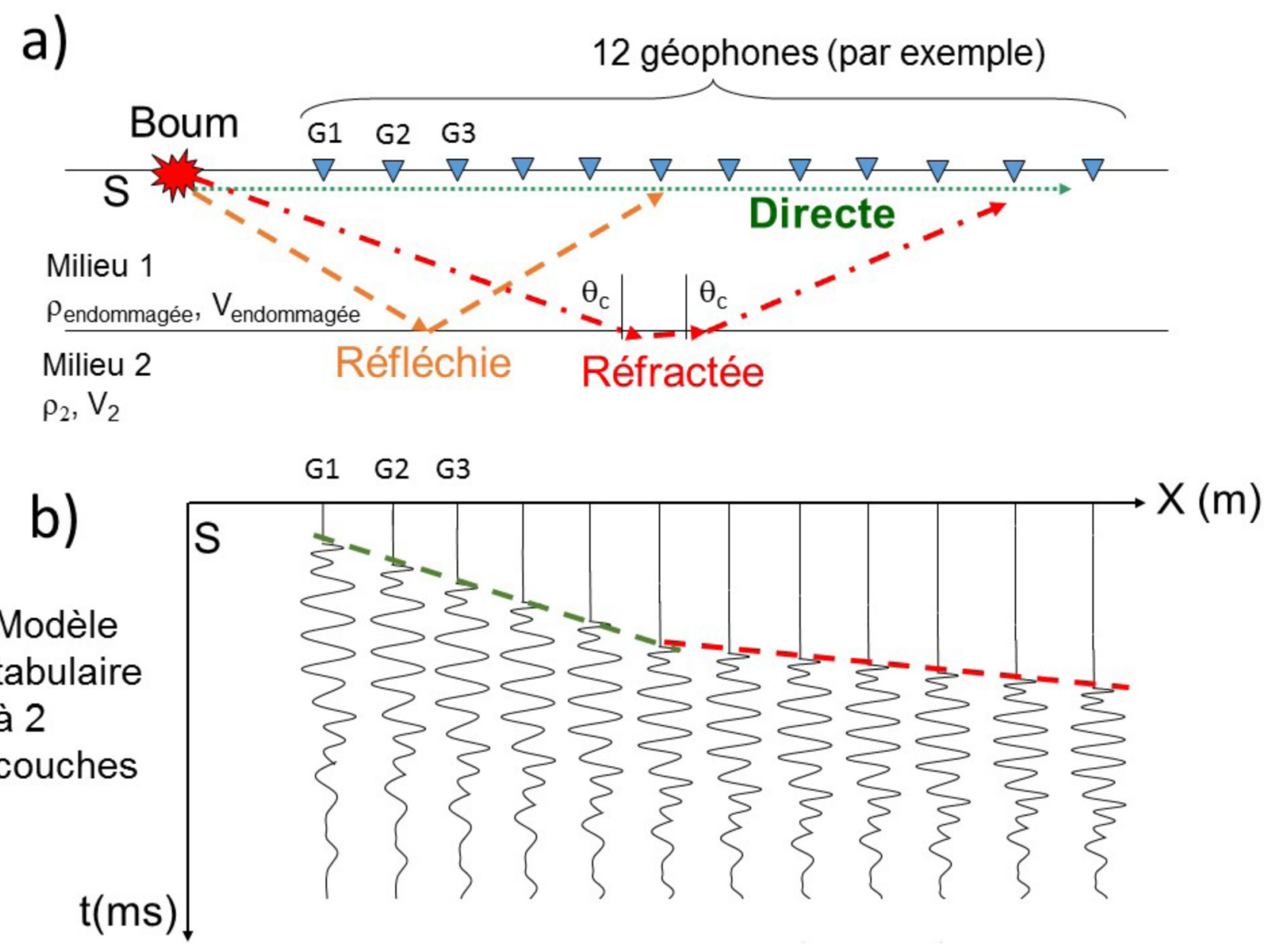

Fig. 4. Sismique réfraction : a : modèle simple à deux terrains de la zone endommagée ; $\mathrm{b}:$ dromochronique correspondante au modèle. Fig. 4. Seismic refraction: a: two-layer model of damaged zone; $b$ : time-distance graph of the corresponding model.

\subsection{Sismique réfraction}

En sismique réfraction, comme pour toutes les méthodes de prospection sismique de sub-surface, une onde élastique est générée artificiellement grâce à une sollicitation mécanique brève à la surface du sol (coup de marteau ou de fusil, explosion d'une faible charge de dynamite, etc.). Les déplacements des particules du sol résultant de cette sollicitation se propagent de proche en proche dans le milieu en formant des surfaces concentriques de rayon croissant appelées surfaces d'ondes. Ce processus de propagation est souvent représenté par des rayons (ou rais) sismiques perpendiculaires aux surfaces d'ondes

Sur le modèle tabulaire simplifié de zone endommagée (milieu 1) de la Figure 4a, trois rayons sismiques sont dessinés et correspondent à trois ondes : l'onde $\mathrm{P}$ directe, l'onde $\mathrm{P}$ qui se réfléchit à l'interface des milieux 1 et 2 de vitesses et de masse volumique différentes, et enfin l'onde P réfractée se propageant sur la même interface à la vitesse $V_{2}$, qui apparaît loin de la source sismique. La méthode de sismique réfraction consiste alors à placer des récepteurs appelés géophones à la surface du sol (12 géophones dans l'exemple de la Fig. 3a) au voisinage de la source $\mathrm{S}$ et à " pointer » les premières arrivées sur les enregistrements. Les pointés des premières arrivées sont alors reportés sur un graphique sur lequel la position des géophones est indiquée en abscisse et le temps pointé en ordonnée (Fig. 4b). Les points de ce graphique s'organisent souvent en segments de droite (droites en pointillés sur la Fig. 4b) appelés dromochroniques ou hodochrones dont les pentes sont ici dans cet exemple les inverses des vitesses des ondes des deux milieux (zone endommagée et zone saine). En sismique réfraction, l'interprétation consiste alors à calculer, à partir des dromochroniques, la profondeur des interfaces à l'aplomb de chacun des géophones et les vitesses des milieux considérés.

À notre connaissance et en considérant la problématique de cet article, la méthode de sismique réfraction a été mise en œuvre pour la première fois avec succès dans la galerie EST96 de la station expérimentale de Tournemire de l'Institut de radioprotection et de sûreté nucléaire (IRSN) (Magnin et al., 2008 ; Gélis et al., 2010). Le dispositif consistait en des profils sur radier de 30 accéléromètres espacés de $10 \mathrm{~cm}$ sur une longueur de $2,9 \mathrm{~m}$ avec huit tirs. Compte tenu de la longueur réduite du profil en comparaison avec celles des dispositifs classiques du génie-civil et de la faible profondeur des cibles envisagée, une chaîne d'acquisition spécifique et des capteurs particuliers travaillant à des fréquences de plusieurs kilohertz ont été utilisés permettant une incertitude sur les pointés de l'ordre de quelques dizaines de microsecondes (Gélis et al., 2010). 


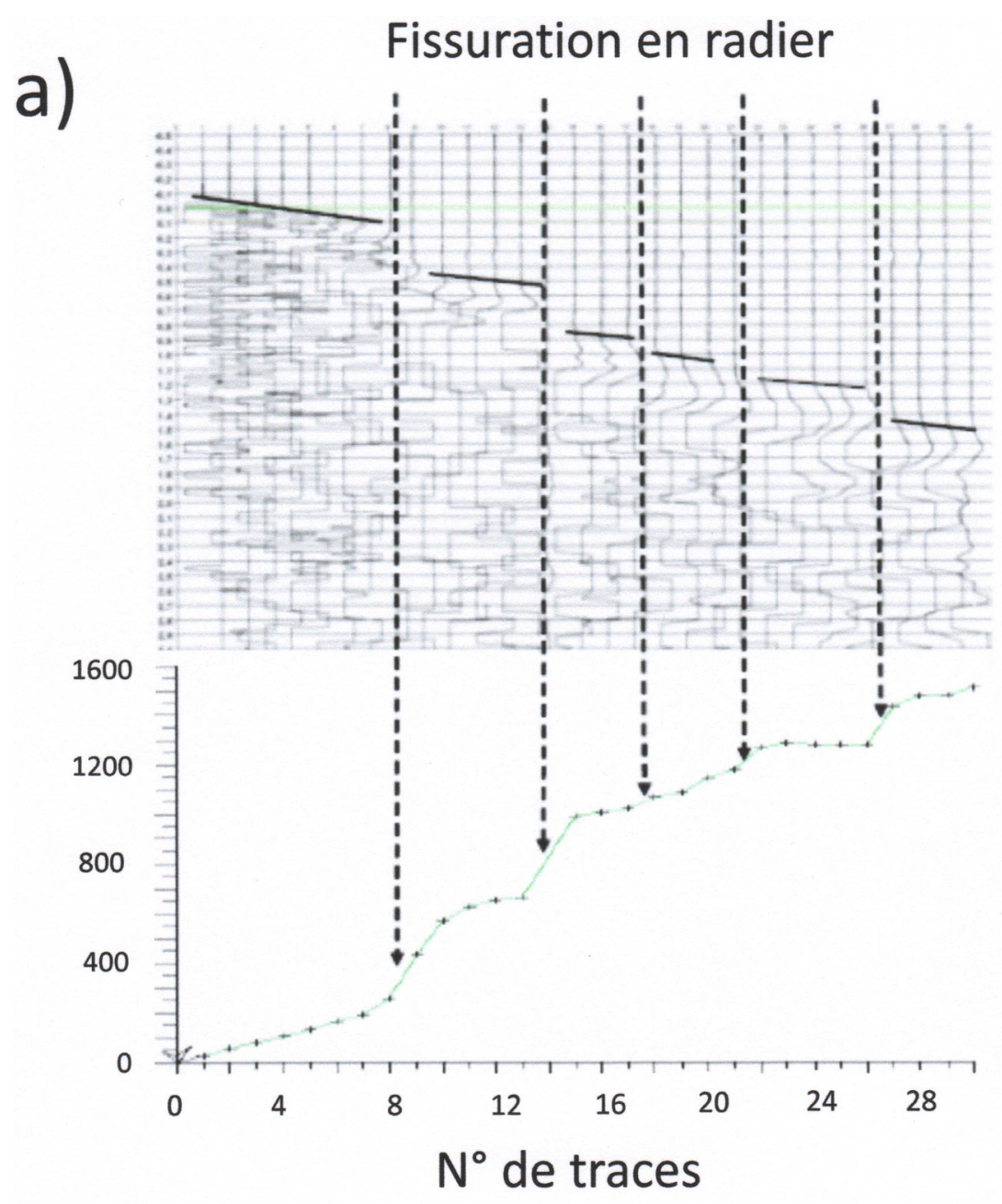

b)

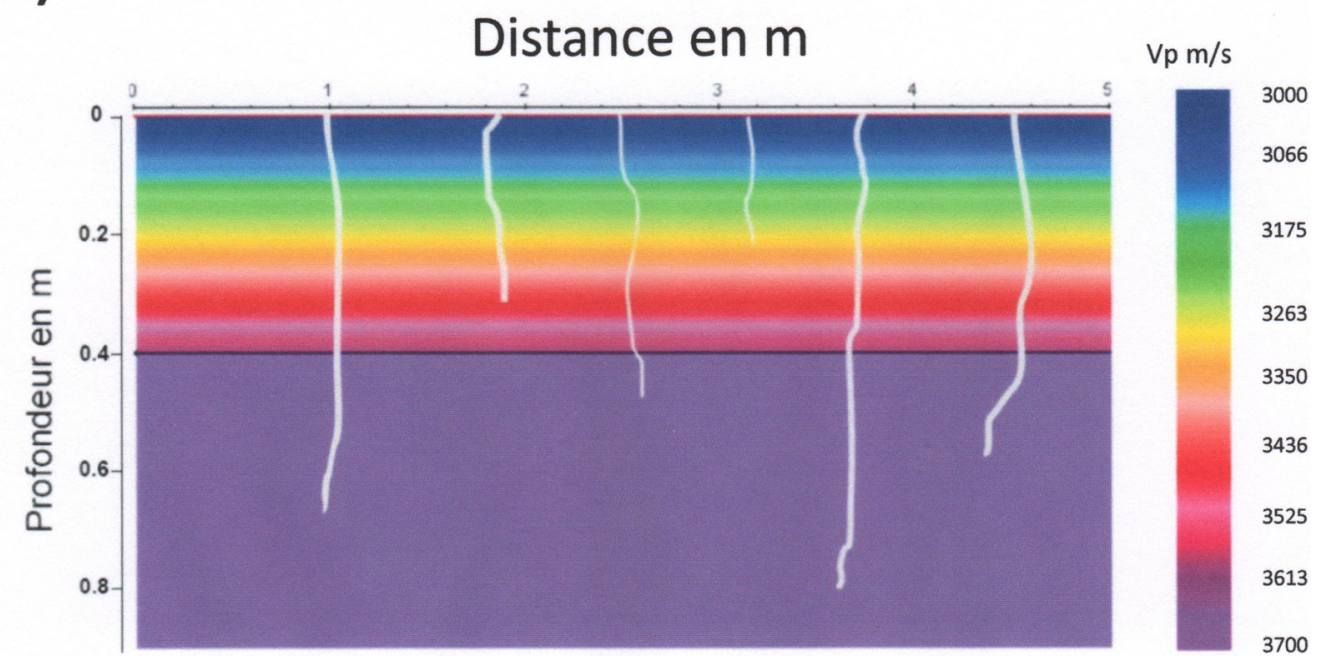

Fig. 5. a : Dromochronique de la campagne de sismique réfraction acquise sur le radier de la galerie EST96 du site de Tournemire. Les « sauts » de la dromochronique indiquent la position de fractures sur le radier ; b : modèle de zone endommagée sur le radier de galerie obtenu à partir de la sismique réfraction.

Fig. 5. a: Time-distance graph of the refraction seismic survey performed on the floor of EST96 gallery at the Tournemire site. The jumps of the graph indicate the location of cracks on the floor; $b$ : model of damaged zone on the gallery floor obtained from seismic refraction. 
Les discontinuités observées de la dromochronique obtenue ont été clairement associées aux fissures perpendiculaires au profil sismique réalisé (Fig. 5a). En outre, l'interprétation générale des profils sismiques a permis de proposer le modèle de vitesses suivant : les vitesses en onde $\mathrm{P}$ dans la zone endommagée évoluent de 2900 à $3100 \mathrm{~m} . \mathrm{s}^{-1}$ pour atteindre les valeurs de 3700 à $3800 \mathrm{~m} . \mathrm{s}^{-1}$ dans la roche saine.

Malgré sa relative simplicité de mise en œuvre et d'interprétation (comparativement aux autres méthodes sismiques), la sismique réfraction souffre d'un inconvénient qu'il ne faudrait pas occulter : une onde réfractée est générée à l'interface de deux milieux si, et seulement si, la vitesse du milieu sous-jacent (milieu 2 ou roche saine sur l'exemple de la Fig. 4a) est supérieure à celle du milieu sus-jacent, le moins profond (zone endommagée sur la Fig. 4a). Or, dans le contexte d'une galerie présentant un soutènement rigide en béton par exemple, il est fort probable que la vitesse de la zone endommagée (sous le soutènement en béton) soit inférieure à celle du béton : la sismique réfraction ne peut être appliquée et il faut alors recourir à d'autres méthodes comme celle de l'analyse de la dispersion des ondes de surfaces.

\subsection{Analyse de la dispersion des ondes de surface}

Comme mentionné précédemment, une source sismique à la surface du sol génère, en plus des ondes de volume, des ondes de surface qui restent confinées près de la surface du sol : ce sont les ondes de Love et les ondes de Rayleigh. Les ondes de Rayleigh qui jouent un rôle central dans la méthode présentée ici sont associées à des déplacements elliptiques des particules du sol qui restent localisées dans le plan vertical de propagation (Mari et al., 1998). En comparaison avec les ondes de volume, l'amplitude des ondes de surface décroît moins rapidement au cours de leur propagation : elles sont donc plus facilement détectables loin de la source sismique et ne nécessitent pas de source très énergétique. En outre, si le milieu traversé est stratifié, ou plus généralement verticalement hétérogène, ces ondes deviennent dispersives : la vitesse de phase des ondes est dépendante de la fréquence de propagation. Différents modes de propagation rentrent alors en jeu. Enfin, les équations de propagation de telles ondes permettent également de montrer que leur vitesse de phase dépend principalement de la vitesse de l'onde de cisaillement (ou onde S) et donc du module de cisaillement G (Mari et al., 1998 ; Bodet, 2005 ; Bodet et al., 2005).

Par conséquent, la relative simplicité de détection des ondes de surface dans l'enregistrement sismique et les relations existantes entre leur vitesse et des propriétés pétrophysiques du géomatériau (masse volumique, module de cisaillement) en font un outil de reconnaissance potentiellement intéressant. La méthode MASW (Multichanel Analysis of Surface Waves) associée à une source vibratoire est certainement l'approche la plus pertinente pour la problématique qui nous intéresse (Lagarde et al., 2006 ; Magnin et al., 2008 ; Gélis et al., 2010 ; Leparoux et al., 2012). En pratique, la méthode consiste à enregistrer les arrivées sismiques en ondes de surface sur une série de récepteurs alignés à la source, espacés avec un pas régulier et installés sur la paroi d'une galerie. L'analyse de ces enregistrements notamment par des méthodes de transformation de champ d'onde dont l'exposé dépasse largement le cadre de cet article permet alors d'obtenir un diagramme de dispersion (diagramme de vitesses de phase versus fréquence pour les différents modes de propagation) qui autorise l'évaluation du caractère dispersif des ondes de surface mises en jeu. Dans une dernière étape, les courbes de dispersions associées aux différents modes de propagation (en pratique, les courbes reliant les maxima du diagramme de dispersion) peuvent être inversées pour obtenir un profil de vitesses d'onde $\mathrm{S}$ en fonction de la profondeur (Bodet, 2005 ; Bodet et al., 2005 ; Leparoux et al., 2012).

Les résultats donnés sur les Figures $6 \mathrm{a}$ et $6 \mathrm{~b}$ sont issus d'une campagne de mesures réalisée sur le radier en béton d'une galerie du site de Tournemire (Gélis et al., 2010 ; Leparoux et al., 2012). Le dispositif associé présentait 93 géophones espacés de $10 \mathrm{~cm}$, sur une longueur totale de 9,2 $\mathrm{m}$ et une source vibratoire de type sweep (une sinusoïde de fréquence linéairement croissante de 150 à $1800 \mathrm{~Hz}$ sur une durée de 3,5 s). La géométrie de ce dispositif, c'est-à-dire la distance inter-géophone et la longueur du dispositif, a été déterminée en fonction de la profondeur d'investigation recherchée ( $2 \mathrm{~m}$ environ) qui définissait alors la gamme de longueurs d'ondes du signal enregistré.

La Figure 6a montre une interprétation du diagramme de dispersion expérimental correspondant à cette campagne sur laquelle le mode fondamental et le premier mode supérieur ont été indiqués. La Figure $6 \mathrm{~b}$ donne le résultat de l'inversion de deux courbes de dispersion associées à ces deux premiers modes, en termes de profil de vitesses d'ondes $\mathrm{S}$, fonction de la profondeur. Cette inversion a permis de localiser la profondeur maximale supposée de la zone endommagée sous le radier en béton estimée entre 0,79 et $0,98 \mathrm{~m}$ dans cette étude de cas.

\subsection{Diagraphie sismique}

Les diagraphies sont des méthodes géophysiques mises en œuvre au moyen de sondes dites diagraphiques disposées à l'intérieur d'un forage (Lagabrielle, 2007) (Fig. 3c). Ces sondes disposent d'une source sismique et souvent de plusieurs capteurs-récepteurs (généralement des accéléromètres). Les ondes élastiques générées se propagent entre la source et les capteurs-récepteurs dans le rocher au voisinage de la paroi. Le volume investigué par ces méthodes est donc du même ordre grandeur que celui du forage : ce sont donc des méthodes plutôt locales. En outre, comme elles nécessitent un forage, elles sont donc en partie invasives et l'endommagement induit par le forage reste problématique.

Ces techniques permettent toutefois de mesurer in situ une ou des « lenteurs » sismiques (i.e., les inverses des vitesses sismiques) avec une résolution verticale élevée (de l'ordre de quelques centimètres). Elles sont donc complémentaires des autres techniques sismiques présentées dans cet article qui, elles, permettent d'obtenir une information spatialisée sur des volumes plus importants mais avec une plus faible résolution spatiale.

La diagraphie sismique est, à notre connaissance, la première méthode sismique à avoir été utilisée pour l'étude de la zone endommagée en contexte argileux (Schuster et al., 2001) (Tab. 2). Ce sont certainement les résultats acquis par cette étude qui ont permis d'impulser tous les travaux utilisant la propagation d'ondes élastiques dans les roches argileuses. 


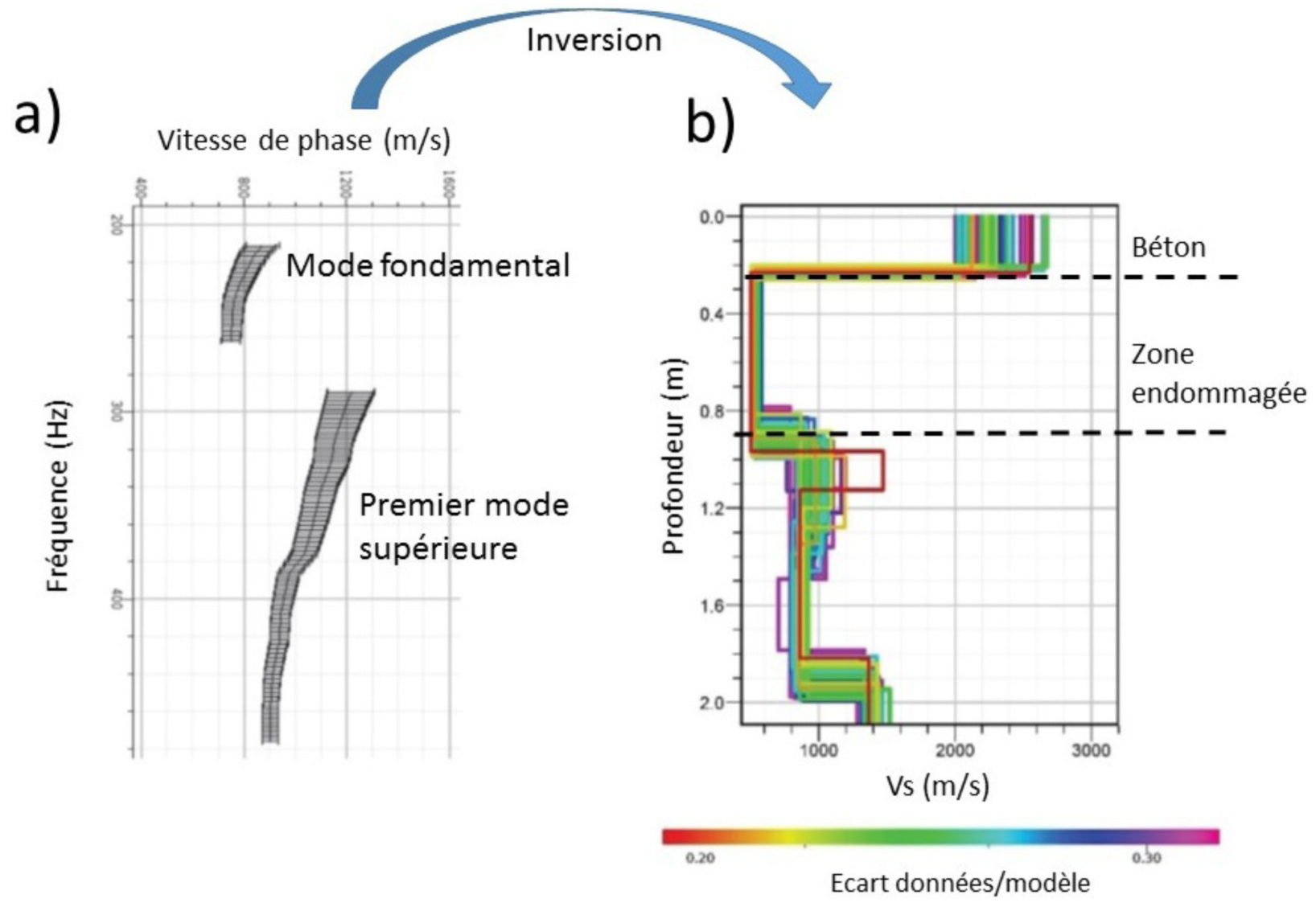

Fig. 6. a : Analyse de la dispersion des ondes de surface. Identification du mode fondamental et du premier mode supérieur à partir d'un diagramme de dispersion expérimental obtenu sur le site de Tournemire ; b : modèle de vitesse d'onde $\mathrm{S}$ issu de l'inversion. Les épaisseurs du radier béton et de la zone endommagée sont indiquées (modifié de Leparoux et al., 2012).

Fig. 6. a: Multichanel Analysis of Surface Waves method. Identification of the fundamental mode and the first higher mode from an experimental dispersion diagram acquired at the Tournemire site; $b$ : model of $S$-wave velocity obtained from inversion. The thicknesses of the concrete layer and the damaged zone are indicated (modified from Leparoux et al., 2012).

Les deux études majeures utilisant la diagraphie sismique sont présentées de manière synthétique dans le Tableau 2. Pour la problématique qui nous intéresse, les travaux de Balland et Renaud (2009) sont probablement les plus aboutis. Ces auteurs ont développé une sonde microsismique permettant de faire varier la distance source-récepteur (et donc le volume d'investigation autour $\mathrm{du}$ forage) et la position angulaire des récepteurs autour de l'axe de la sonde. Cette sonde peut en outre fonctionner dans un forage sec, condition intéressante en contexte argileux. Les degrés de liberté des liaisons récepteurs-sonde et l'utilisation d'un code d'inversion adaptée à cette technique offrent la possibilité d'obtenir une image 3D de champs de vitesse autour du forage. Cette technique a été employée dans le laboratoire souterrain de Meuse/Haute-Marne et met en évidence une zone endommagée anisotrope s'étendant à 0,35 rayon de forage (Balland et Renaud, 2009).

\subsection{Méthodes sismiques « puits à puits »}

Dans les méthodes sismiques de «puits à puits», l'émission des ondes mécaniques est faite dans un forage émetteur, la réception de ces mêmes ondes dans un autre forage équipé de récepteurs (géophones ou hydrophones lorsque le forage est rempli d'eau) (Mari et al., 1998). L'existence de forages souligne que ces techniques sont donc sensiblement invasives. Comme pour beaucoup de méthodes sismiques ici décrites, elles supposent que la propagation des ondes peut être représentée par un rayon (ou rai) sismique (voir Sect. 2.1).

Dans le contexte de la caractérisation in situ de la zone endommagée autour d'une galerie, deux méthodes sismiques « puits à puits » sont utilisées. La première consiste à analyser les vitesses estimées à partir des temps de trajet entre émetteurs et récepteurs (leurs positions étant parfaitement connues) des deux forages associés (Balland et al., 2009 ; Le Gonidec et al., 2012). La seconde méthode est la tomographie sismique qui produit une image (carte 2D) d'une propriété mécanique (une vitesse ou un coefficient d'atténuation) limitée à la région interforage (Manukyan et al., 2012 ; Balland et al., 2013). Cette image est obtenue par des techniques d'inversion utilisant les temps de trajet et/ou les amplitudes des ondes les premières arrivées sur les récepteurs (Côte et Lagabrielle, 1986).

En pratique, les zones de faible vitesse sismique mises en évidence par ces méthodes sont souvent identifiées à un zonage de l'endommagement autour de la galerie (Fig. 7). Toutefois, on gardera à l'esprit que cette identification n'est pas toujours justifiée pour toutes les vitesses. Les travaux de Manukyan et al. 
Tableau 2. Résumé des campagnes de diagraphie sismique.

Table 2. Summary of seismic logging surveys.

\begin{tabular}{|c|c|c|c|c|c|}
\hline $\begin{array}{l}\text { Référence } \\
\text { (par ordre } \\
\text { chronologique) }\end{array}$ & Problématique & $\begin{array}{l}\text { Principales } \\
\text { caractéristiques du } \\
\text { dispositif }\end{array}$ & $\begin{array}{l}\text { Principales } \\
\text { caractéristiques de } \\
\text { l'interprétation }\end{array}$ & $\begin{array}{l}\text { Résultats - } \\
\text { Points forts }\end{array}$ & Points faibles \\
\hline
\end{tabular}

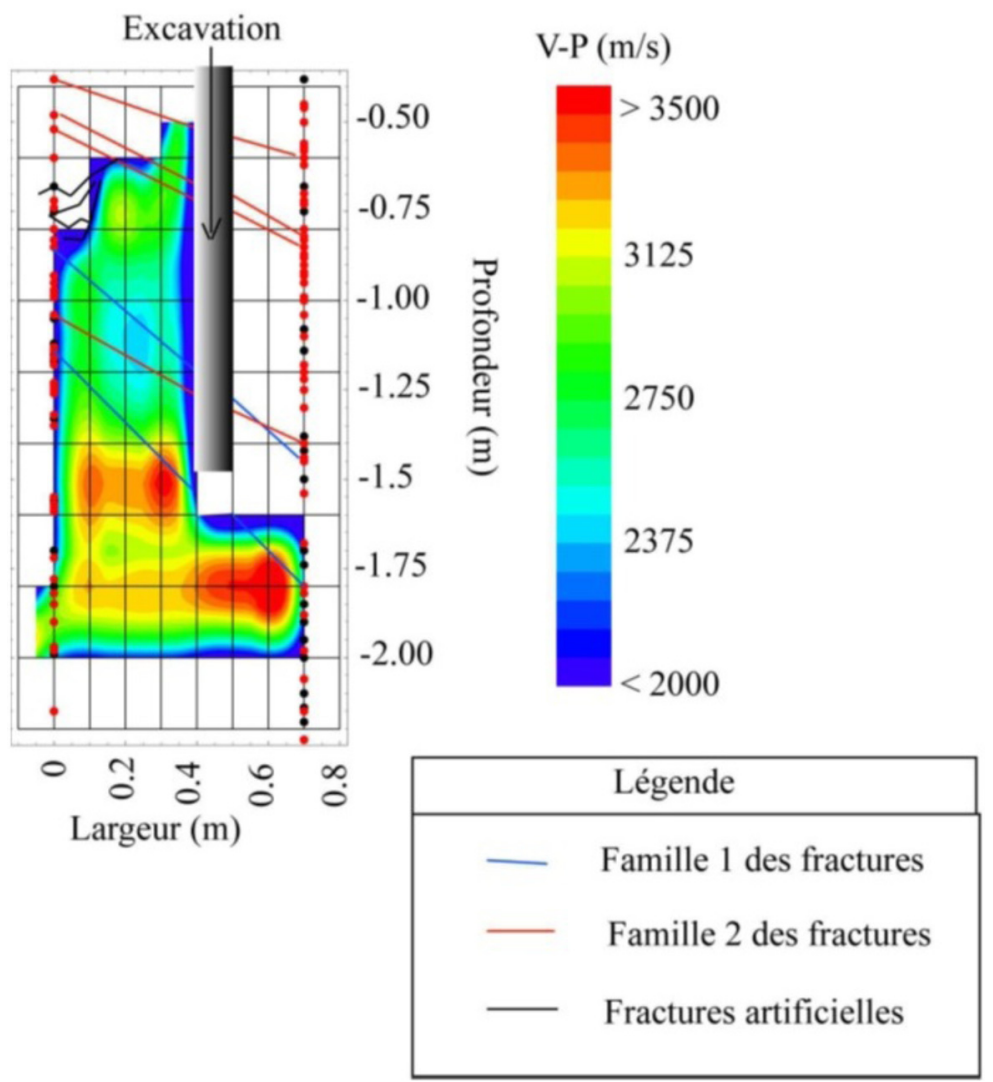

Fig. 7. Carte des vitesses d'ondes $\mathrm{P}$ obtenue par tomographie sismique. La zone de faibles vitesses est associée à des familles fractures indiquées sur la figure (Damaj, 2006).

Fig. 7. P-waves map acquired from seismic tomography. The low-values zone is associated with the presence of groups of fractures (Damaj, 2006). 


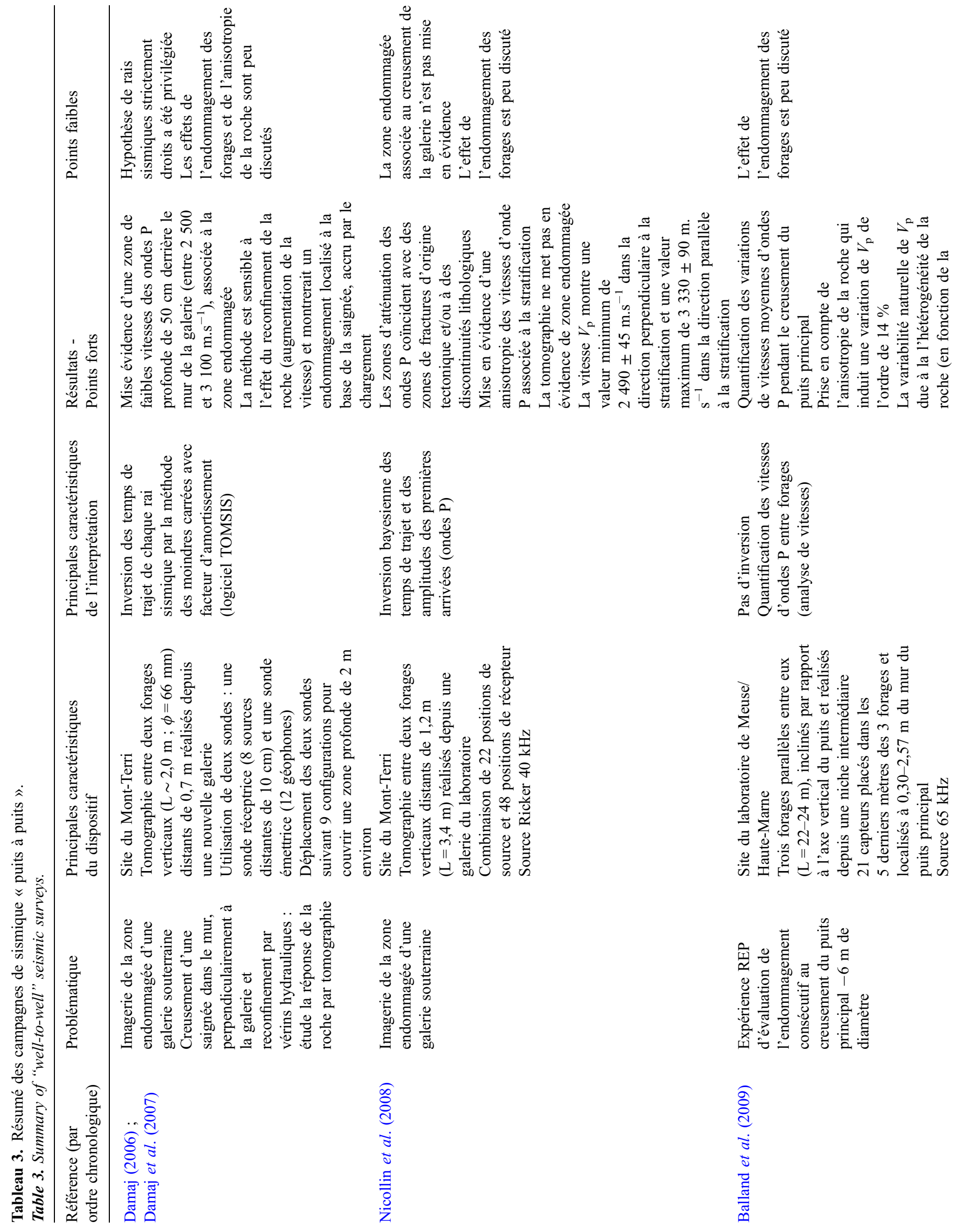




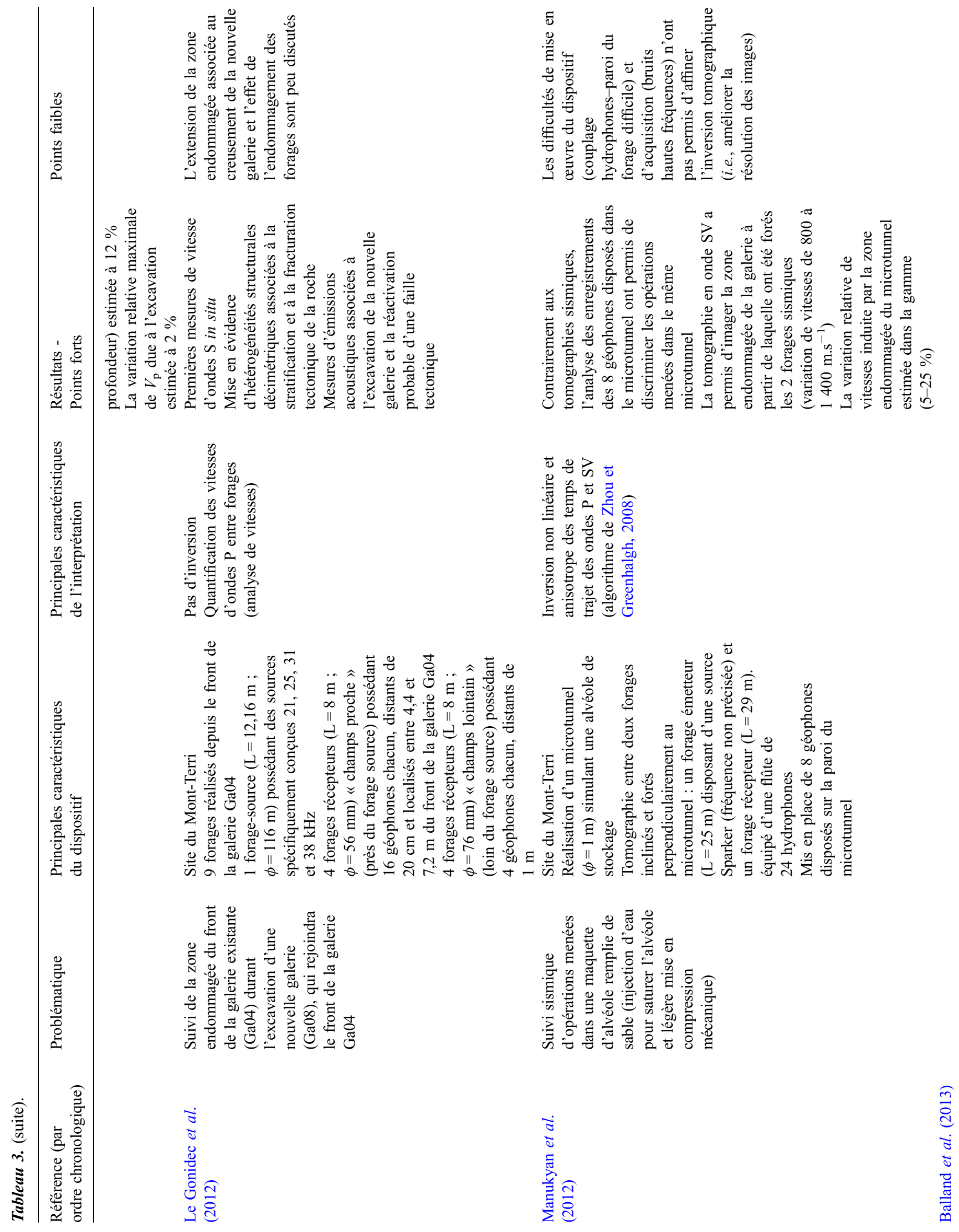


P. Cosenza et al. : Rev. Fr. Geotech. 2017, 148, 4

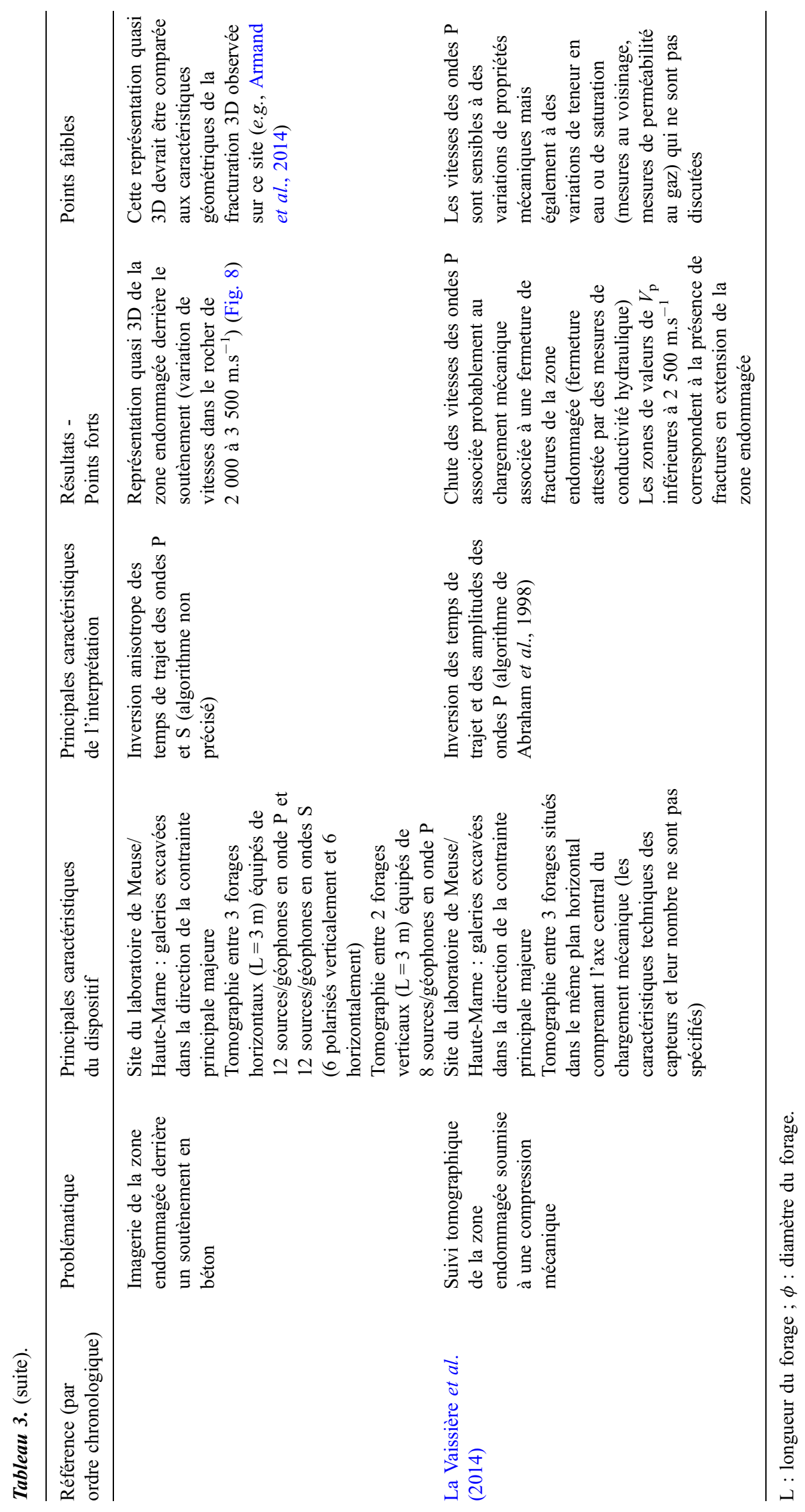

Page 13 de 22 


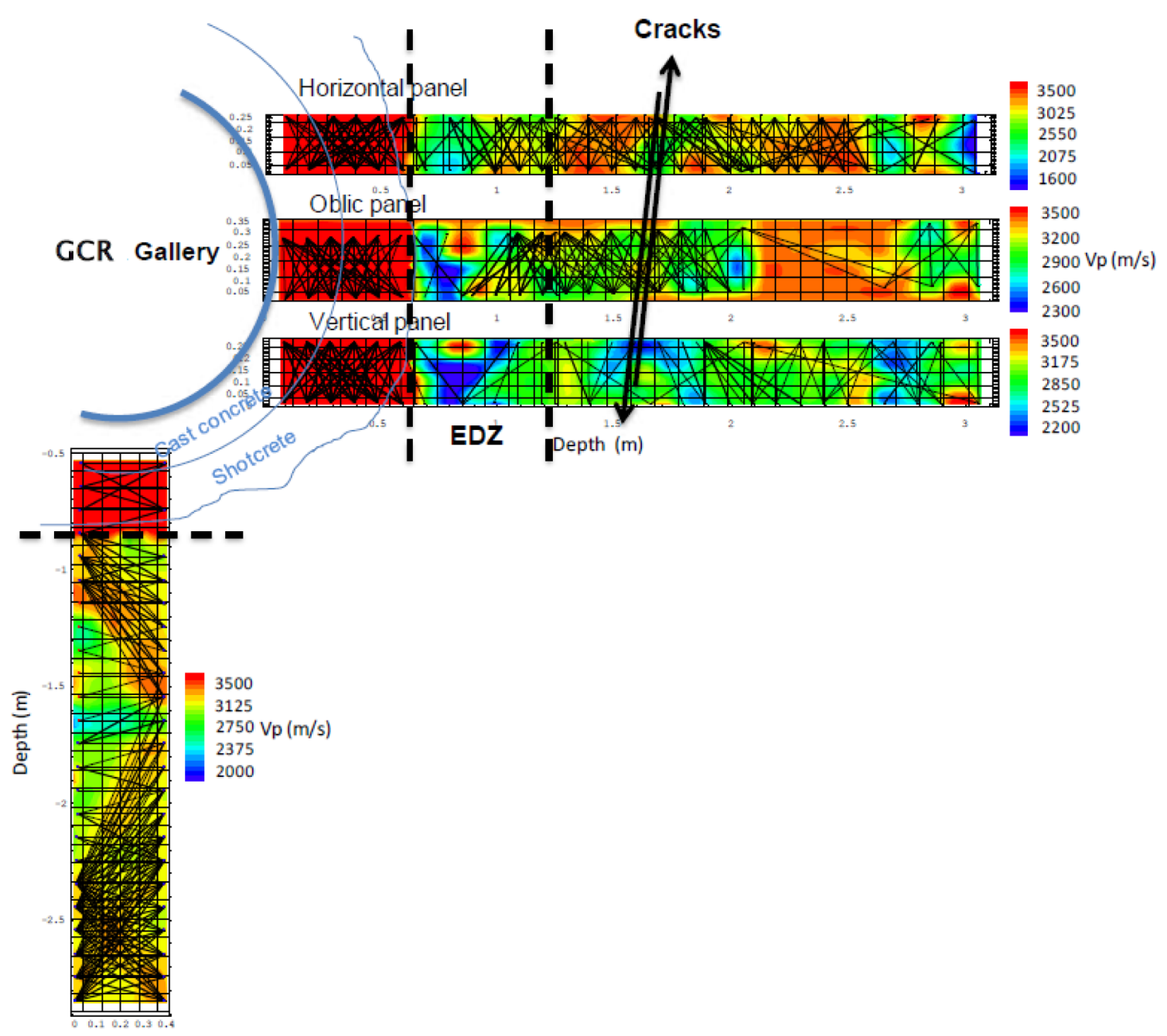

Fig. 8. Tomographies sismiques en ondes P dans la galerie GCR du laboratoire souterrain de Meuse/Haute-Marne (Balland et al., 2013). Le soutènement et la zone endommagée (EDZ) sont également indiqués.

Fig. 8. P-waves seismic tomography in the GCR gallery in the underground Meuse/Haute-Marne laboratory (Balland et al., 2013). Lining and excavation damaged zone (EDZ) area are also indicated.

(2012) illustrent bien cet aspect : c'est la tomographie en onde S, et non celle en onde $\mathrm{P}$, qui aura permis d'imager le mieux la zone endommagée d'une galerie du site du Mont-Terri.

Les principaux travaux utilisant des méthodes sismiques de «puits à puits » sont résumés dans le Tableau 3 dans lequel les forces et les faiblesses de chaque étude sont présentées. Ce tableau permet de constater que la méthodologie a su évoluer rapidement avec des techniques utilisées pour une caractérisation «statique » de la zone endommagée (Nicollin et al., 2008) vers des approches permettant un suivi temporel (Manukyan et al., 2012 ; La Vaissière et al., 2014) et des représentations quasi $3 \mathrm{D}$ de la zone endommagée (Balland et al., 2013) (Fig. 8). Les développements méthodologiques récents de ces techniques suggèrent que les suivis tomographiques pourraient être significativement améliorés en inversant directement les temps d'arrivée pour obtenir des tomographies en mode time-lapse c'est-à-dire en comparant les résultats des inversions obtenus pour chaque pas de temps (i.e., tomographie en différence de temps d'arrivée, en anglais differential travel time tomography, e.g.Maurer et al., 2014).

\section{Tomographie électrique}

La méthode électrique la plus utilisée pour la caractérisation de la zone endommagée autour des ouvrages est la tomographie de résistivité électrique $2 \mathrm{D}$. Cette méthode vise à obtenir une distribution 2D, i.e., une « image », de la résistivité électrique dans le rocher.

En pratique, une résistivité électrique est mesurée à l'aide de quatre électrodes métalliques. Un courant continu, $I$, est injecté dans le terrain via une paire d'électrodes et la différence de potentiel électrique, $\Delta V$, résultante est mesurée sur une autre paire d'électrodes. La résistivité électrique est alors calculée à partir de la résistance, $\Delta V / I$, en multipliant cette dernière par un coefficient géométrique qui dépend des positions relatives des quatre électrodes et de la géométrie du terrain (Fig. 9a). Lorsque les quatre électrodes sont alignées, avec le même écart entre électrodes, le dispositif électrique mis en jeu se nomme dispositif Wenner.

Dans le cas de la tomographie électrique, on utilise généralement un système multi-électrodes composé d'une série d'électrodes reliée à un résistivimètre via un multiplexer. Un 


\section{a) Mesure de résistivité}

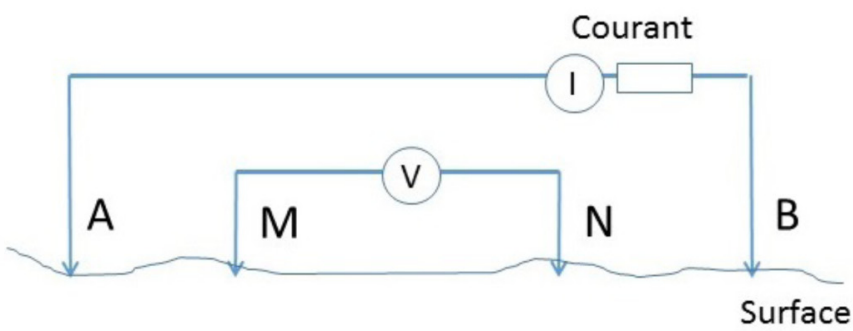

$$
\rho=K \frac{\Delta V}{I}=K \frac{V_{M}-V_{N}}{I_{A->B}}
$$

\section{b) Tomographie électrique 2D (panneau électrique)}

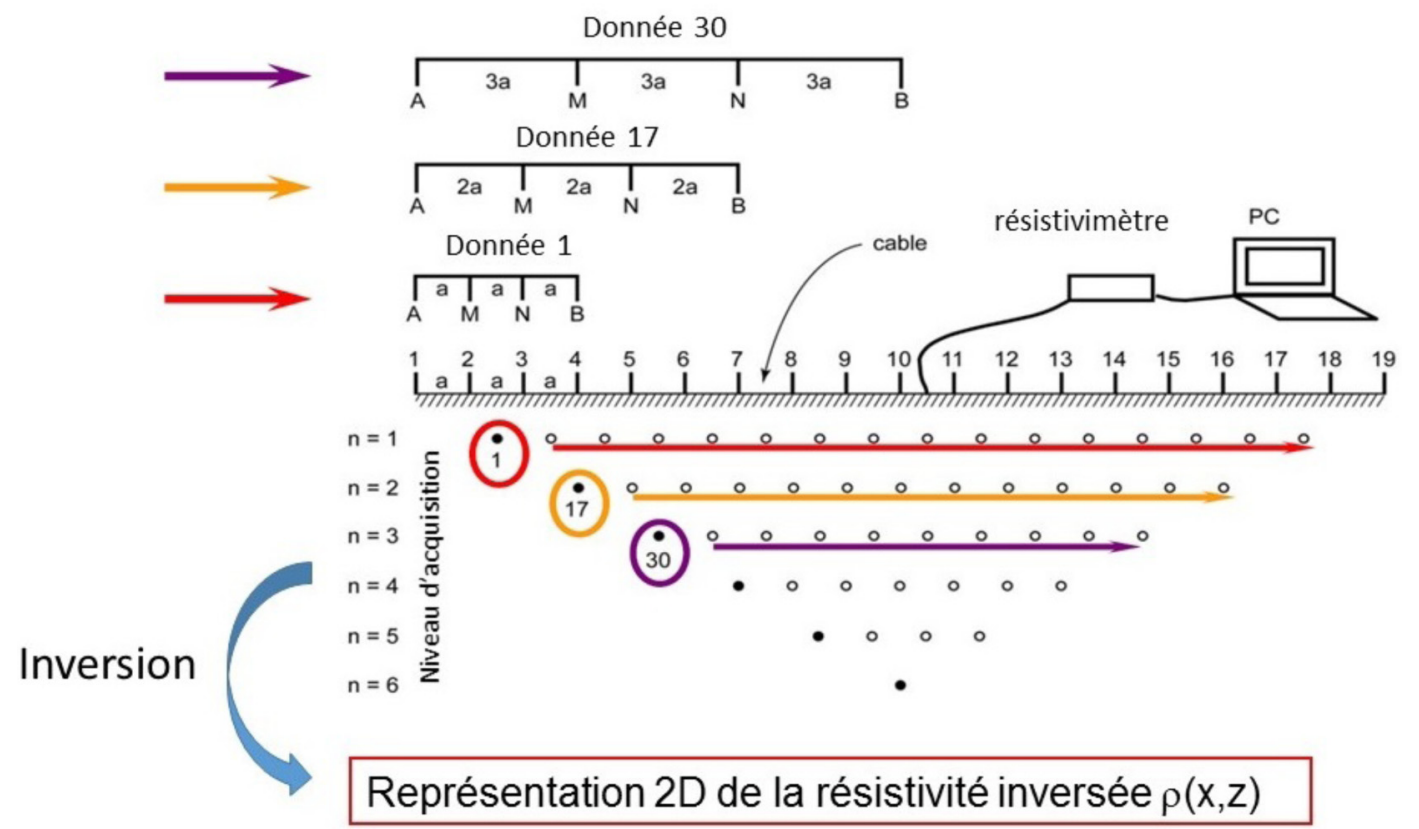

Fig. 9. a : Principe de la mesure de résistivité ; b : principe de la tomographie électrique 2D (exemple : dispositif Wenner).

Fig. 9. a: Resistivity measurements principle; $b$ : $2 D$ electrical tomography (example: Wenner array).

courant est injecté par deux électrodes sources et le potentiel résultant est mesuré sur une ou plusieurs paires d'électrodes réceptrices. Cette procédure est répétée plusieurs dizaines, voire centaines, de fois en considérant plusieurs combinaisons de paires d'électrodes sources et de paires d'électrodes réceptrices suivant une séquence prédéfinie. L'ensemble de résistivités dites «apparentes » ainsi obtenues est alors généralement inversé par un logiciel souvent commercial (par exemple, Res2DInv, Loke et Barker, 1995, utilisant une approche de type moindres carrés). Le résultat de cette inversion est une distribution 2D (une image) de résistivité électrique « inversée » (Fig. 9b).
La Figure 10 présente un exemple d'images obtenues sur le radier en béton de la galerie EST03 sur le site de Tournemire (Okay, 2011 ; Okay et al., 2013). Sur ces images, il est possible de distinguer le radier en béton (milieu 1, épaisseur d'environ $30 \mathrm{~cm}$; résistivité d'environ $180 \mathrm{Ohm} . \mathrm{m}$ ), l'argilite désaturée et endommagée (milieu 2, d'environ $70 \mathrm{~cm}$; résistivité entre 500 et 1600 Ohm.m) et l'argilite non endommagée (milieu 3, résistivité entre 10 et $60 \mathrm{Ohm} . \mathrm{m})$. On remarquera que la position de la zone endommagée identifiée sur ces modèles de résistivité est cohérente avec celle obtenue par analyse de la dispersion des ondes de surface sur le même site (Fig. 6b). Toutefois, comparativement aux méthodes sismi- 


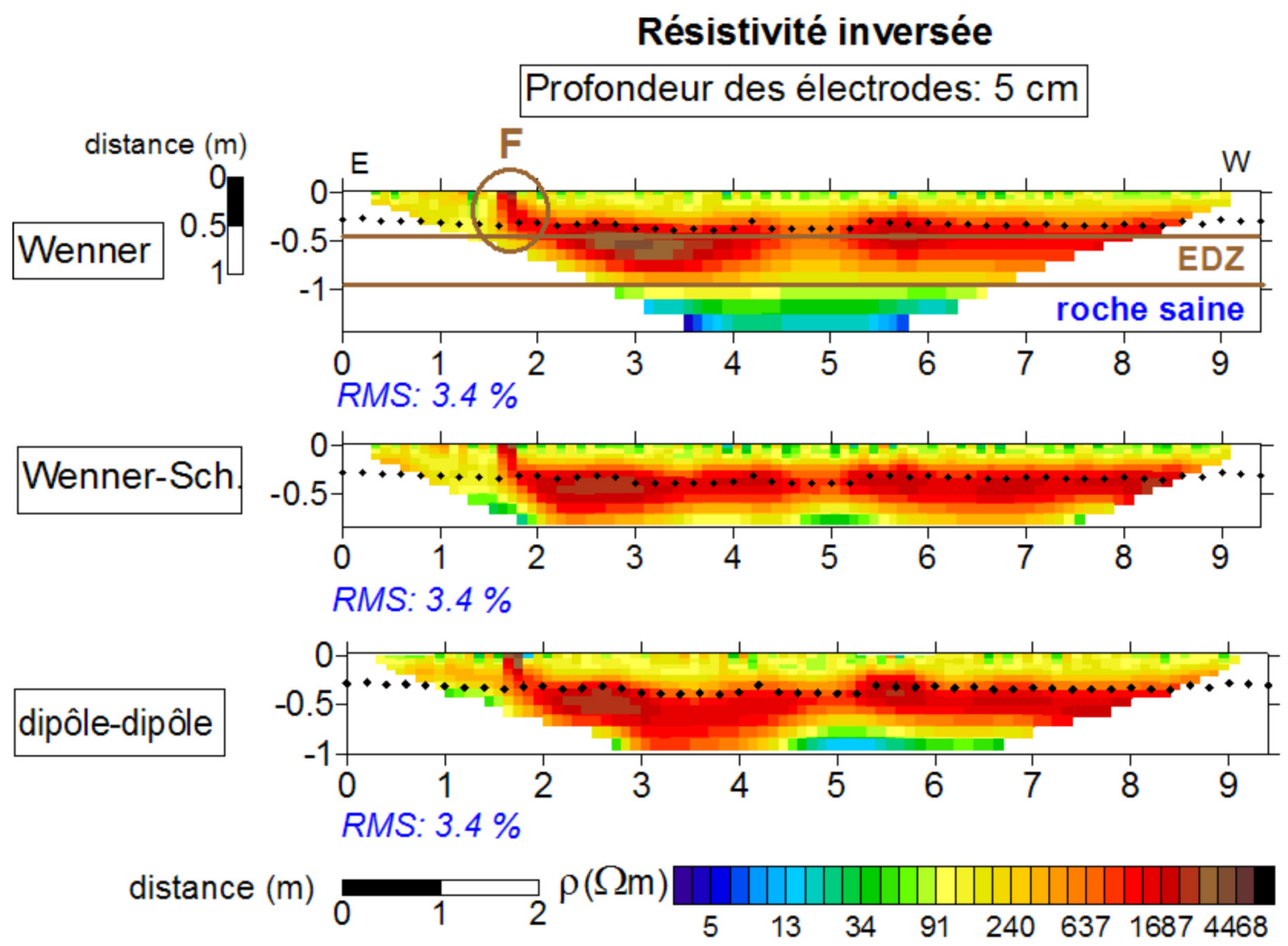

Fig. 10. Exemple de campagne de tomographie électrique 2D. Modèles de résistivités inversées suivant trois configurations (Wenner, WennerSchlumberger et dipôle-dipôle) obtenues sur le radier béton de la galerie EST03 du site de Tournemire (EDZ : zone endommagée ; F : position d'une fracture d'origine tectonique observée sur le radier). Les lignes en pointillés indiquent la position de l'interface béton-zone endommagée (Okay, 2011).

Fig. 10. Example of 2D electrical resistivity tomography. Models of inverted resistivity following three arrays (Wenner, Wenner-Schlumberger et dipôle-dipôle) obtained on the concrete floor of the EST03 gallery at the Tournemire site (EDZ: excavated damaged zone; F: location of tectonic fault observed on the floor). The dotted lines indicate the location of the concrete/EDZ interface (Okay, 2011).

ques, l'identification de la frontière entre roche endommagée et roche saine par tomographie électrique apparaît généralement moins précise.

Les principaux travaux utilisant la tomographie électrique sont résumés dans le Tableau 4 dans lequel les forces et les faiblesses de chaque étude sont présentées. Ce tableau permet de formuler les constats suivants. D'une part, pour que la tomographie électrique soit pleinement opérationnelle dans notre contexte, il est nécessaire que le processus d'inversion prenne explicitement en compte la géométrie circulaire d'une galerie souterraine ; cette fonctionnalité est absente dans la plupart des logiciels commerciaux. Cette nécessité impose donc des développements numériques spécifiques. D’autre part, la tomographie électrique est souvent utilisée comme une méthode complémentaire aux méthodes sismiques afin d'identifier plus spécifiquement la zone désaturée de la roche (Mayor et al., 2007). En effet, il est nécessaire ici de rappeler que les ions de la solution interstitielle sont les principaux vecteurs du courant électrique mis en jeu dans cette méthode : la résistivité électrique est donc sensible aux variations de teneur en eau porale du matériau (voir aussi Fig. 2). Enfin, lorsque la tomographie de résistivité électrique est couplée à une tomographie en polarisation provoquée qui s'obtient souvent avec le même équipement géophysique, elle permet de discriminer des fractures à remplissage de minéraux polarisables (pyrite, argiles) (Okay et al., 2013, 2014). D’une manière simple, la polarisation provoquée consiste à mesurer le retard de la réponse (en différence de potentiel électrique) d'un matériau soumis à un champ électrique externe. Ce retard se traduit dans le domaine fréquentiel par un déphasage entre courant injecté et différence de potentiel électrique mesurée ; c'est pourquoi, il est souvent associé à un effet capacitif apparent du milieu.

\section{Méthodes optiques de corrélation}

Les méthodes optiques de corrélation sont a priori intéressantes pour la caractérisation quantitative de la fracturation présente en paroi des ouvrages souterrains. En effet, ce sont des méthodes sans contact permettant de spatialiser directement des champs cinématiques (déplacements et déformations) et de quantifier l'ouverture et la fermeture de fissures. Les méthodes optiques de corrélation 


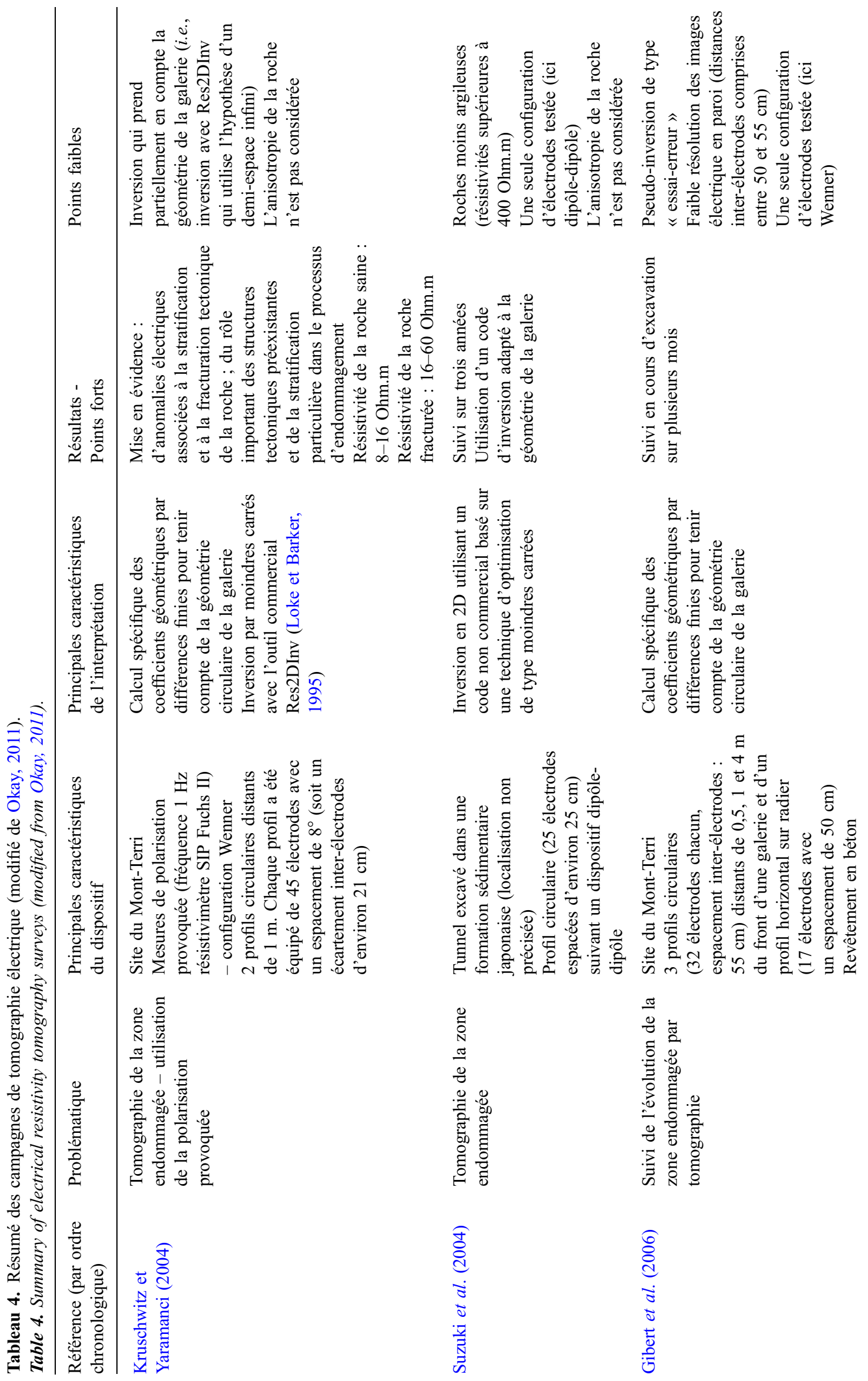


P. Cosenza et al. : Rev. Fr. Geotech. 2017, 148, 4

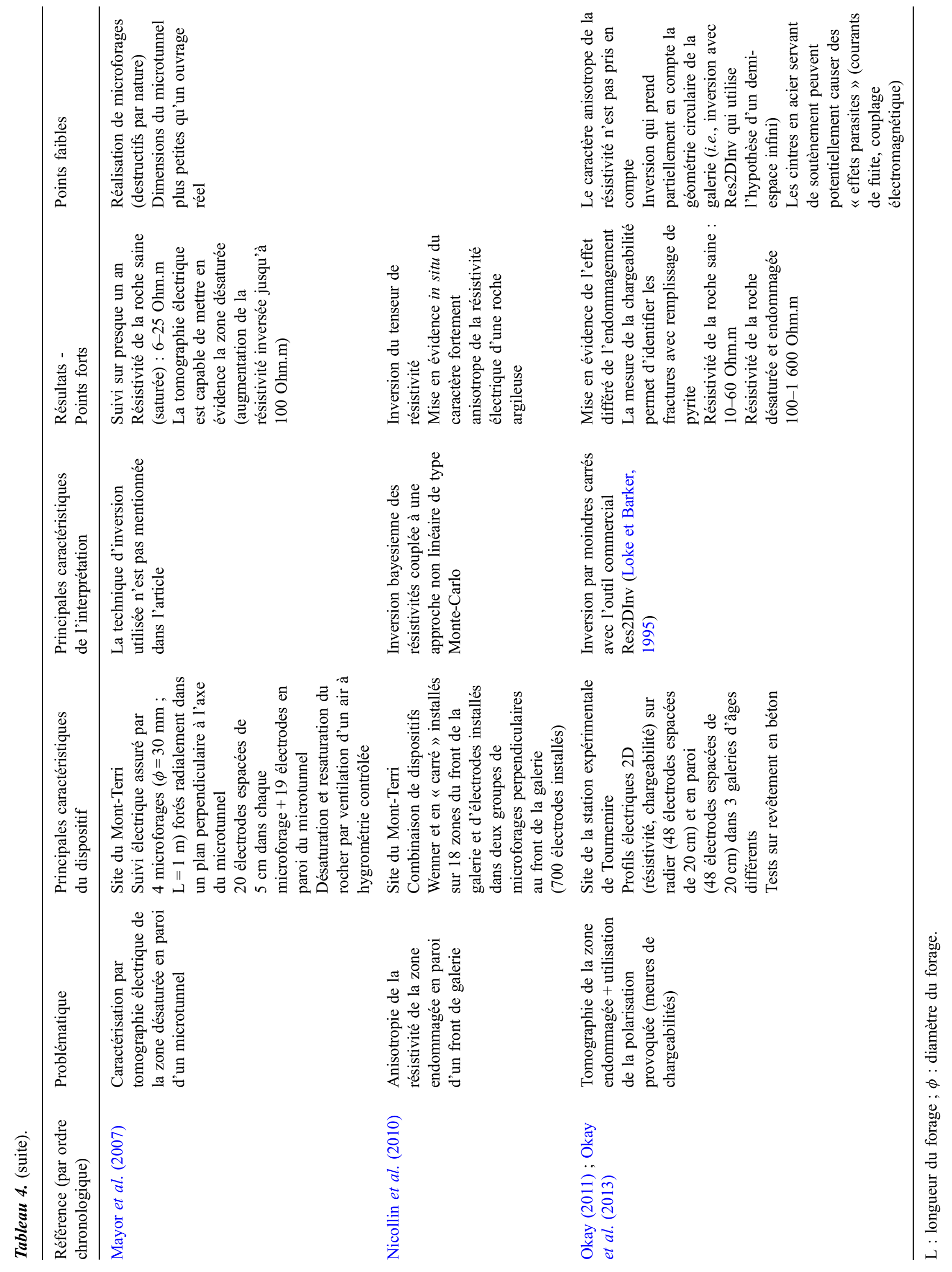

Page 18 de 22 


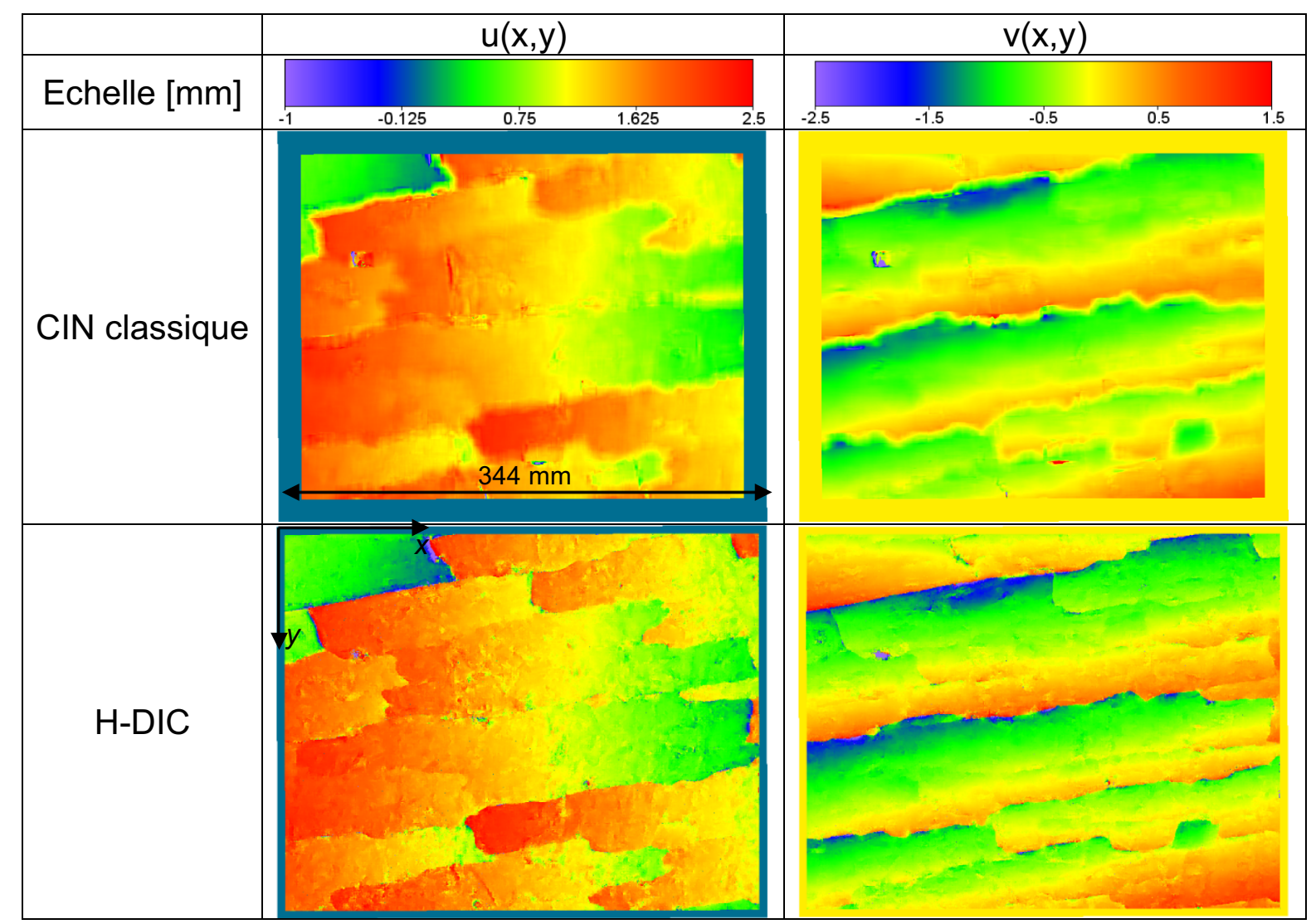

Fig. 11. Champs des déplacements ( $u$ : composante horizontale ; $v$ : composante verticale) obtenus sur le site de Tournemire par méthode de corrélation d'image numérique (CIN) classique et par la méthode H-DIC (heaviside-based digital image correlation) (Hedan et al., 2016).

Fig. 11. Displacements field (u: horizontal component; v: vertical component) obtained from a classical digital image correlation (DIC) method and from a H-DIC (heaviside-based digital image correlation) method at the Tournemire site (Hedan et al., 2016).

consistent à enregistrer des images numériques d'une surface au cours d'une transformation physique, afin d'obtenir des champs de déplacement de la surface filmée. Les méthodes de corrélation se basent alors sur la comparaison de sousdomaines de niveaux de gris entre deux images. La corrélation entre ces sous-domaines est calculée à partir d'une fonction de corrélation basée sur une transformation simple permettant de mesurer les déplacements plans $(u, v)$ avec une précision inférieure au pixel (par exemple, Hedan et al., 2012, 2014).

À notre connaissance, ces méthodes ont été appliquées pour la première fois en galerie souterraine sur le site de Tournemire (Hedan et al., 2014). Le dispositif expérimental mis en place dans la galerie EST96 comprenait une zone d'étude $\left(344 \times 275 \mathrm{~mm}^{2}\right)$ située sur le front de la galerie. Des images furent acquises toutes les 30 minutes en utilisant une caméra CMOS IDS $\mu$ eye $\left(1280 \times 1024\right.$ pixels $\left.^{2}\right)$ fixée à un cintre de soutènement et équipée d'un objectif $50 \mathrm{~mm}$ et d'un projecteur lumineux de $400 \mathrm{~W}$. Un micro-ordinateur contrôlait simultanément l'acquisition des images et l'allumage du projecteur lors de l'acquisition des images. Des données climatiques (hygrométrie et température) ont été enregistrées toutes les 15 minutes à l'aide de capteurs placés à une distance de $2 \mathrm{~m}$ de la zone d'étude. La configuration du dispositif expérimental a permis une résolution spatiale et la taille d'un sous-domaine d'environ $269 \mu \mathrm{m}$ et $10,8 \mathrm{~mm}$, respectivement.
Les composantes des déplacements plans de la Figure 11 ont été obtenues par un algorithme de corrélation classique (corrélation d'images numériques [CIN] classique) et un algorithme spécialement développé dans lequel la transformation physique prend en compte les discontinuités (heaviside based digital image correlation [H-DIC], Valle et al., 2015). Les discontinuités des champs de déplacement de la Figure 11 permettent de localiser des fissures mais également de quantifier l'ouverture et la fermeture de ces mêmes fissures (Hedan et al., 2016). Un exemple d'une telle quantification est donné sur la Figure 12 correspondant à un suivi pluriannuel de l'ouverture et la fermeture d'une fissure.

En outre, il est important de mentionner que des champs de déformations planes peuvent être également calculés à partir des champs de déplacement. Toutefois, ces champs de déformations ne peuvent être estimés que sur des périodes limitées (inférieures à trois mois) (Hedan et al., 2014) à cause d'une évolution trop forte du contraste de niveaux de gris des images associées à la poussière présente dans la galerie.

Plus récemment, ces techniques ont été utilisées pour suivre l'évolution temporelle des déformations d'une paroi d'une galerie souterraine (laboratoire souterrain Meuse/Haute-Marne) consécutivement à la dépose de cintres de soutènement (Hedan et al., 2016). L'originalité de l'expérimentation a consisté notamment à mesurer la composante hors-plan du champ de 


\section{Ouverture $(\mathrm{mm})$}

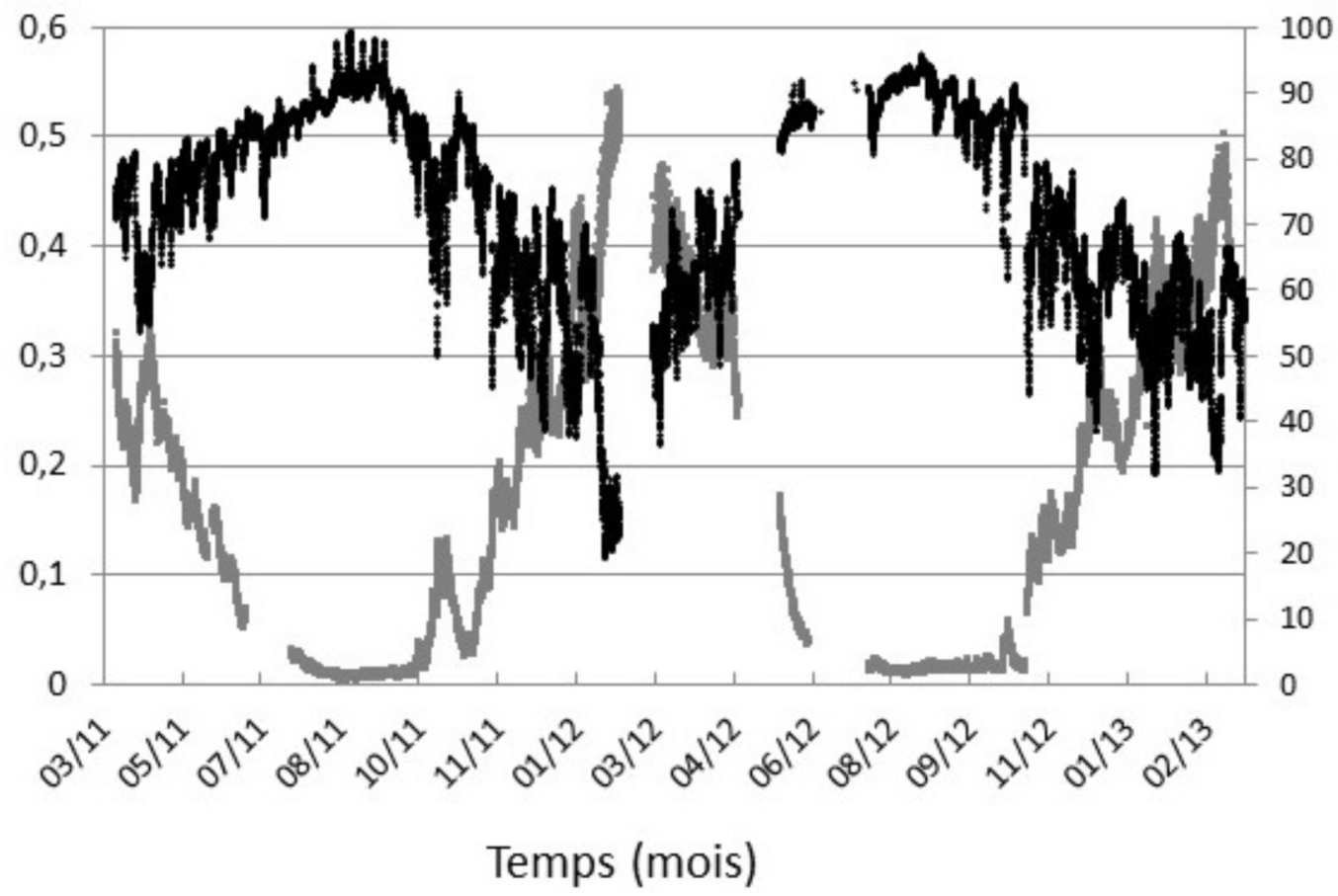

Fig. 12. Suivi sur deux années de l'ouverture d'une fissure d'origine hydrique (ligne grise) corrélée avec l'évolution de l'humidité relative (ligne noire) de la galerie EST96 du site de Tournemire.

Fig. 12. Monitoring over two years of a crack aperture (grey line) correlated with the relative humidity change (black line) in the EST96 gallery of the Tournemire site.

déplacement de la paroi (convergence) par stéréo-corrélation en utilisant deux caméras filmant la même zone d'étude. Ce développement méthodologique a ainsi permis d'assurer un suivi 3D des fissures à la surface de la paroi de la galerie.

\section{Conclusion}

Nous avons présenté un état de l'art des méthodologies non destructives utilisées pour la caractérisation et le suivi de la zone endommagée des ouvrages excavés dans les roches argileuses. Cet état de l'art, résumé dans le Tableau 5, permet in fine d'identifier trois familles de méthodes. Une première famille, comprenant la sismique réfraction et l'analyse de la dispersion des ondes de surface, permet d'avoir rapidement et avec un dispositif plutôt léger une quantification de l'épaisseur de la zone endommagée. Une deuxième famille de méthodes relevant encore de la géophysique (méthodes sismiques « puits à puits » et tomographie électrique) permet d'obtenir une représentation $2 \mathrm{D}$, voire $3 \mathrm{D}$, de cette zone endommagée. Les développements méthodologiques récents relatifs à ces méthodes permettent d'envisager à court terme un suivi 4D en promouvant une inversion de type time-lapse des données acquises. Ces méthodes sont toutefois plus lourdes à mettre en œuvre et l'interprétation des données nécessite des temps de calcul importants et des outils numériques très spécifiques et délicats à manipuler pour un non-géophysicien. Les méthodes optiques de corrélation relèvent d'une troisième famille de méthodes. Elles ont surtout l'avantage d'obtenir directement et avec une très haute résolution des grandeurs mécaniques (cinématiques) associées à la fissuration de surface.

Le Tableau 5 met également en évidence l'intérêt de coupler ces méthodes dans au moins trois situations : le couplage de la tomographie sismique et de la tomographie électrique pour le suivi de l'endommagement et de la désaturation de la roche à l'échelle de la galerie, le couplage de la tomographie sismique et de la diagraphie sismique pour le suivi de l'endommagement de la roche à l'échelle de la galerie en contrôlant l'endommagement des forages géophysiques, et enfin le couplage de techniques de tomographie et de méthodes optiques de corrélation pour l'étude de l'initiation et la propagation de la fissuration en paroi. Ce dernier couplage constitue une perspective naturelle des travaux menés actuellement in situ sur la zone endommagée des ouvrages souterrains. Ces études montrent également que le caractère anisotrope (isotrope transverse) de la roche argileuse est encore rarement pris en compte dans l'interprétation des données. L'anisotropie de la roche argileuse et ses effets géomécaniques doivent continuer à faire l'objet de recherches tant sur le plan théorique que sur le terrain, en galerie souterraine.

Remerciements. Une partie des travaux présentés dans cet article de revue est issue d'une collaboration fructueuse avec des collègues qui sont ici vivement remerciées : J. Cabrera, C. Camerlynck, N. Florsch, A. Ghorbani, J. Morel, G. Okay, A. Revil. 
Tableau 5. Synthèse des apports, des avantages et des inconvénients des méthodes utilisées pour la caractérisation non destructive de la zone endommagée des galeries souterraines excavées dans les roches argileuses.

Table 5. Synthesis of contributions, advantages and drawbacks of methods used for the non-destructive characterization of the damaged zone of underground galleries excavated in clay rocks.

\begin{tabular}{|c|c|c|c|}
\hline Méthodes & Contributions & Avantages & Inconvénients \\
\hline Sismique réfraction & Épaisseur de la zone endommagée & Légèreté du dispositif d'acquisition & $\begin{array}{l}\text { À éviter si radier en béton } \\
\text { Modèle 1D }\end{array}$ \\
\hline $\begin{array}{l}\text { Analyse de la dispersion } \\
\text { des ondes de surface }\end{array}$ & $\begin{array}{l}\text { Épaisseur de la zone endommagée } \\
\text { Modèle 1D de la zone endommagée }\end{array}$ & Légèreté du dispositif d'acquisition & $\begin{array}{l}\text { Inversion difficile si dispositif } \\
\text { circulaire en paroi de la galerie }\end{array}$ \\
\hline Diagraphie sismique & $\begin{array}{l}\text { Représentation } 2 \mathrm{D} / 3 \mathrm{D} \text { de la zone } \\
\text { endommagée }\end{array}$ & $\begin{array}{l}\text { Très bonne résolution verticale } \\
\text { Très bonne identification de la } \\
\text { frontière entre roche endommagée } \\
\text { et roche saine } \\
\text { Quantification de l'anisotropie } \\
\text { des vitesses }\end{array}$ & $\begin{array}{l}\text { Réalisation d'un forage } \\
\text { Données localisées autour du forage } \\
\text { de la sonde diagraphique }\end{array}$ \\
\hline $\begin{array}{l}\text { Méthodes sismiques } \\
\text { "puits à puits » }\end{array}$ & $\begin{array}{l}\text { Représentation } 2 \mathrm{D} / 3 \mathrm{D} \text { de la zone } \\
\text { endommagée }\end{array}$ & $\begin{array}{l}\text { Bonne résolution } \\
\text { Quantification de l'anisotropie } \\
\text { des vitesses }\end{array}$ & $\begin{array}{l}\text { Réalisation de forages } \\
\text { Inversion spécifique pour prendre en } \\
\text { compte la géométrie circulaire de la } \\
\text { galerie }\end{array}$ \\
\hline $\begin{array}{l}\text { Méthodes optiques de } \\
\text { corrélation }\end{array}$ & $\begin{array}{l}\text { Quantification de la cinématique des } \\
\text { fractures-fissures en des parois }\end{array}$ & $\begin{array}{l}\text { Méthodes sans contact } \\
\text { Simplicité de la mise en œuvre } \\
\text { Grandeurs cinématiques spatialisées } \\
\text { à très haute résolution }\end{array}$ & $\begin{array}{l}\text { Données de «surface » } \\
\text { Développement d'algorithmes } \\
\text { spécifiques pour prendre en compte } \\
\text { les fissures }\end{array}$ \\
\hline
\end{tabular}

\section{Références}

Abraham O, Ben Slimane K, Cote P. 1998. Factoring anisotropy into iterative geometric reconstruction algorithms for seismic tomography. Int J Rock Mech Min Sci 35: 31-41.

AGAP-Qualité. 1992. Géophysique appliquée. Code de bonne pratique. Disponible sur http://www.agapqualite.org/code-debonne-pratique.html, dernière consultation 2016/09/09.

Armand G, Leveau F, Nussbaum C, et al. 2014. Geometry and properties of the excavation-induced fractures at the Meuse/HauteMarne URL drifts. Rock Mech Rock Eng 47: 21-41.

Balland C, Renaud V. 2009. High-resolution velocity field imaging around a borehole: Excavation-damaged zone characterization. Geophysics 74: E223-E232.

Balland C, Morel J, Armand G, Pettitt W. 2009. Ultrasonic velocity survey in Callovo-Oxfordian argillaceous rock during shaft excavation. Int J Rock Mech Min Sci 46: 69-79.

Balland C, Morel J, Souley M. 2013. Ultrasonic sounding and monitoring of the excavation damaged zone in relation with drift support. In: ASEG Extended Abstracts 2013. Australian Society of Exploration Geophysicists (ASEG), pp. 1-4.

Baechler S, Lavanchy JM, Armand G, Cruchaudet M. 2011. Characterisation of the hydraulic properties within the EDZ around drifts at level $-490 \mathrm{~m}$ of the Meuse/Haute-Marne URL: a methodology for consistent interpretation of hydraulic tests. Phys Chem Earth, Parts A/B/C 36: 1922-1931.

Blümling P, Bernier F, Lebon P, Martin CD. 2007. The excavation damaged zone in clay formations time-dependent behaviour and influence on performance assessment. Phys Chem Earth, Parts A/B/ C 32: 588-599.

Bodet L. 2005. Limites théoriques et expérimentales de l'interprétation de la dispersion des ondes de Rayleigh : apport de la modélisation numérique et physique. Mémoire de thèse de doctorat, Nantes (France).

Bodet L, van Wijk K, Bitri A, et al. 2005. Surface-wave inversion limitations from laser-Doppler physical modeling. J Environ Eng Geophys 10: 151-162.

Bossart P, Meier PM, Moeri A, Trick T, Mayor JC. 2002. Geological and hydraulic characterisation of the excavation disturbed zone in the Opalinus clay of the Mont Terri Rock Laboratory. Eng Geol 66: 19-38.

Cosenza P, Ghorbani A, Florsch N, Revil A. 2007. Effects of drying on the low-frequency electrical properties of Tournemire argillites. Pure Appl Geophys 164: 2043-2066.

Côte P, Lagabrielle R. 1986. La tomographie sismique comme méthode de reconnaissance détaillée du sous-sol - exemple d'application au contrôle d'injection. Rev Fr Geotech 36: 47-53. 
Damaj J. 2006. Auscultation et surveillance des perturbations hydromécaniques d'ouvrages souterrains par méthodes ultrasonores. Mémoire de thèse de doctorat. Vandœuvre-les-Nancy, INPL (France).

Damaj J, Balland C, Armand G, Verdel T, Amitrano D, Homand F. 2007. Velocity survey of an excavation damaged zone: influence of excavation and reloading. Geol Soc Lond, Spec Publ 284: 41-55.

Delle Piane C, Almqvist BS, MacRae CM, Torpy A, Mory AJ, Dewhurst DN. 2015. Texture and diagenesis of Ordovician shale from the Canning Basin, Western Australia: implications for elastic anisotropy and geomechanical properties. Mar Pet Geol 59: 56-71.

Gélis C, Cabrera J, Barnichon JD, et al. 2010. Caractérisation de l'EDZ par méthodes sismiques et ultrasoniques. In : Journées nationales de géotechnique et de géologie de l'ingénieur infrastructures, développement durable et énergie " (JNGG 2010), Laboratoire 3S-R. Grenoble, pp. 811-818.

Ghorbani A. 2007. Contribution au développement de la résistivité complexe et à ses applications en environnement. Mémoire de thèse de doctorat. Université Pierre-et-Marie-Curie, Paris (France).

Ghorbani A, Zamora M, Cosenza P. 2009. Effects of desiccation on the elastic wave velocities of clay-rocks. Int J Rock Mech Min Sci 46: 1267-1272.

Gibert D, Nicollin F, Kergosien B, et al. 2006. Electrical tomography monitoring of the excavation damaged zone of the Gallery 04 in the Mont Terri rock laboratory: field experiments, modelling, and relationship with structural geology. Appl Clay Sci 33: 21-34.

Hedan S, Cosenza P, Valle V, Dudoignon P, Fauchille AL, Cabrera J. 2012. Investigation of the damage induced by desiccation and heating of Tournemire argillite using digital image correlation. Int $J$ Rock Mech Min Sci 51: 64-75.

Hedan S, Fauchille AL, Valle V, Cabrera J, Cosenza P. 2014. One-year monitoring of desiccation cracks in Tournemire argillite using digital image correlation. Int J Rock Mech Min Sci 68: 22-35.

Hedan S, Valle V, Noiret A, Armand G, Cosenza P. 2016. Suivi des déformations par méthodes optiques en galerie souterraine (URL Meuse/Haute-Marne). In : Journées nationales de géotechnique et de géologie de l'ingénieur, Nancy (France).

Kruschwitz S, Yaramanci U. 2004. Detection and characterization of the disturbed rock zone in claystone with the complex resistivity method. J Appl Geophys 57: 63-79.

La Vaissière R, Morel J, Noiret A, et al. 2014. Excavation-induced fractures network surrounding tunnel: properties and evolution under loading. Geol Soc Lond, Spec Publ 400: 279-291.

La Vaissière R, Armand G, Talandier J. 2015. Gas and water flow in an excavation-induced fracture network around an underground drift: a case study for a radioactive waste repository in clay rock. J Hydrol 521: 141-156.

Lagabrielle R. 1998. Géophysique appliquée au génie civil. Techniques de l'ingénieur, référence C224 v3.

Lagabrielle R. 2007. Diagraphies et géophysique de forage. Techniques de l'ingénieur, C225 v3.

Lagarde J, Abraham O, Laguerre L, Côte P, Piguet J-P, Balland C, et al. 2006. Use of surface waves and seismic refraction for the inspection of circular concrete structures. Cem Concr Compos 28: 337-348.

Le Gonidec Y, Schubnel A, Wassermann J, et al. 2012. Field-scale acoustic investigation of a damaged anisotropic shale during a gallery excavation. Int $J$ Rock Mech Min Sci 51: 136-148.

Leparoux D, Côte P, Gélis C, Cabrera JC. 2012. EDZ characterization with surface wave analysis: experimental and numerical study for defining feasibility in the context of the Tournemire Platform (France). Near Surf Geophys 10: 401-411.
Loke MH, Barker RD. 1995. Least-squares deconvolution of apparent resistivity pseudosections. Geophysics 60: 1682-1690.

Magnin O, Côte P, Leparoux D, Ben Slimane K, Cabrera-Nunez J. 2008. Investigation géophysique pour caractériser l'endommagement autour d'ouvrages souterrains. In : Journées nationales de géotechnique et de géologie de l'ingénieur : insertion des grands ouvrages dans leur environnement, Nantes (France).

Manukyan E, Maurer H, Marelli S, Greenhalgh SA, Green AG. 2012. Seismic monitoring of radioactive waste repositories. Geophysics 77: EN73-EN83.

Mari JL, Arens G, Chapellier D, Gaudiani P. 1998. Géophysique de gisement et de génie civil. Rueil-Malmaison : Édition Technip.

Maurer H, Spillmann T, Marschall P. 2014. Monitoring of induced stress changes using differential seismic travel time tomography. In: 2014 SEG Annual Meeting, Society of Exploration Geophysicists.

Mayor JC, Velasco M, García-Siñeriz JL. 2007. Ventilation experiment in the Mont Terri underground laboratory. Phys Chem Earth, Parts A/B/C 32: 616-628.

Nicollin F, Gibert D, Bossart P, Nussbaum C, Guervilly C. 2008. Seismic tomography of the excavation damaged zone of the gallery 04 in the Mont Terri Rock Laboratory. Geophys J Int 172: 226-239.

Nicollin F, Gibert D, Lesparre N, Nussbaum C. 2010. Anisotropy of electrical conductivity of the excavation damaged zone in the Mont Terri Underground Rock Laboratory. Geophys J Int 181: 303-320.

Okay G. 2011. Caractérisation des hétérogénéités texturales et hydriques des géomatériaux argileux par la méthode de polarisation provoquée : application à l'EDZ de la station expérimentale de Tournemire. Mémoire de thèse de doctorat, université Pierre-etMarie-Curie, Paris (France).

Okay G, Cosenza P, Ghorbani A, et al. 2013. Localization and characterization of cracks in clay-rocks using frequency and timedomain induced polarization. Geophys Prospect 61: 134-152.

Okay G, Leroy P, Ghorbani A, et al. 2014. Spectral induced polarization of clay-sand mixtures: experiments and modeling. Geophysics 79: E353-E375.

Pettitt WS, Young RP, Balland C, et al. 2004. Development of the tools and interpretation techniques for ultrasonic surveys to monitor the rock barrier around radioactive waste packages in geological repositories. In: OMNIBUS final technical report, FIKW-2001 - 00202, 5th EURA-TOM Framework Program EC, 2004.

Sarout J, Guéguen Y. 2008. Anisotropy of elastic wave velocities in deformed shales: part 1-Experimental results. Geophysics 73: D75-D79.

Schuster K, Alheid HJ, Böddener D. 2001. Seismic investigation of the excavation damaged zone in Opalinus clay. Eng Geol 61: 189-197.

Suzuki K, Nakata E, Minami M, et al. 2004. Estimation of the zone of excavation disturbance around tunnels, using resistivity and acoustic tomography. Explor Geophys 35: 62-69.

Tsang CF, Bernier F, Davies C. 2005. Geohydromechanical processes in the excavation damaged zone in crystalline rock, rock salt, and indurated and plastic clays - in the context of radioactive waste disposal. Int J Rock Mech Min Sci 42: 109-125.

Valle V, Hedan S, Cosenza P, Fauchille AL, Berdjane M. 2015. Digital image correlation development for the study of materials including multiple crossing cracks. Exp Mech 55: 379-391.

Zhou B, Greenhalgh S. 2008. Non-linear travel time inversion for 3-D seismic tomography in strongly anisotropic media. Geophys $J$ Int 172: $383-394$.

Citation de l'article : Philippe Cosenza, Stephen Hedan, Valéry Valle. Caractérisation non destructive de la zone endommagée des galeries souterraines excavées dans les roches argileuses. Rev. Fr. Geotech. 2017, $148,4$. 